\title{
STABILITY OF LARGE-AMPLITUDE SHOCK PROFILES OF GENERAL RELAXATION SYSTEMS*
}

\author{
CORRADO MASCIA ${ }^{\dagger}$ AND KEVIN ZUMBRUN Z $^{\ddagger}$
}

\begin{abstract}
Building on previous analyses carried out in [24, 27], we establish $L^{1} \cap H^{2} \rightarrow L^{p}$ nonlinear orbital stability, $1 \leq p \leq \infty$, with sharp rates of decay, of large-amplitude Lax-type shock profiles for a general class of relaxation systems that includes most models in common use, under the necessary conditions of strong spectral stability, i.e., stable point spectrum of the linearized operator about the wave, transversality of the profile, and hyperbolic stability of the associated ideal shock. In particular, our results apply to standard moment closure systems, answering a question left open in [24]. The argument combines the basic nonlinear stability argument introduced [24] with an improved "Goodman-style" weighted energy estimate similar to but substantially more delicate than that used in [27] to treat large-amplitude profiles of systems with real viscosity.
\end{abstract}

Key words. relaxation systems, stability of traveling waves

AMS subject classifications. 35L60 (35B30 35B45 35L65 76L05)

1. Introduction. In [24], there was carried out a detailed study of linearized and nonlinear stability of traveling front solutions, or shock profiles

$$
(u, v)(x, t)=(\bar{u}, \bar{v})(x-s t), \quad \lim _{z \rightarrow \pm \infty}(\bar{u}, \bar{v})=\left(u_{ \pm}, v_{ \pm}\right)=\left(u_{ \pm}, v^{*}\left(u_{ \pm}\right)\right)
$$

of relaxation systems

$$
\left\{\begin{array}{l}
u_{t}+f(u, v)_{x}=0 \\
v_{t}+g(u, v)_{x}=q(u, v)
\end{array}\right.
$$

$u, f \in \mathbb{R}^{n}, v, g, q \in \mathbb{R}^{r}$, where

$$
\operatorname{Re} \sigma\left(q_{v}\left(u, v^{*}(u)\right)\right)<0
$$

along a smooth equilibrium manifold $v=v^{*}(u)$ defined by $q\left(u, v^{*}(u)\right)=0$.

The linearized results obtained in [24] are extremely general, and appear to be optimal. However, the nonlinear results are restricted to arbitrary-amplitude profiles of special, discrete kinetic models, defined as systems (1.2) for which $f$ and $g$ are linear, and small-amplitude profiles of general, simultaneously symmetrizable models, defined as systems (1.2) for which there exists a smooth, symmetric positive definite matrix function $A^{0}=A^{0}(u, v)$ for which $A^{0} A$ and $A^{0} Q$ are symmetric, where $A:=\left(d f^{t}, d g^{t}\right)^{t}$ and $Q:=\left(0, d q^{t}\right)^{t}$.

The first result is quite satisfactory, and has important applications to the physically interesting class of systems obtained by discretization of kinetic models such

*K.Z. thanks Instituto per le Applicazioni del Calcolo "M. Picone" (CNR) and European TMR Project "Hyperbolic Systems of Conservation Laws" for their hospitality and for making possible the visit (April 10-May 10, 2000) during which this work was initiated. Research of C.M. was supported in part by European TMR Project "Hyperbolic Systems of Conservation Laws". Research of K.Z. was supported in part by the National Science Foundation under Grants No. DMS-0070765 and DMS-0300487.

†Dipartimento di Matematica "G. Castelnuovo", Università di Roma "La Sapienza", P.le Aldo Moro, 2 - 00185 Roma (ITALY), (mascia mat.uniroma1.it)

$\ddagger$ Department of Mathematics, Indiana University, Bloomington, IN 47405-4301 (USA), (kzumbrunindiana.edu) 
as Boltzmann or Vlasov-Poisson equations. On the other hand, the equally important class of systems obtained by moment closure approximation of kinetic models is not contained in the class of discrete kinetic models, and so the question of largeamplitude stability for these models was left open in [24]; indeed, as discussed in Remark 1.16 of [24], it was not at all clear from the analysis of [24] whether this was a technical artifact or represented a true qualitative difference in behavior between these two types of approximation. Moreover, even the requirement of simultaneous symmetrizability appears to be overly restrictive in the large-amplitude case. As described in [33], many systems of physical interest are simultaneously symmetrizable along the equilibrium manifold $v=v^{*}(u)$, including (standard versions of) both discrete and moment-closure approximations of kinetic models; however, so far as we can see, simultaneous symmetrizability does not typically hold away from equilibrium, in particular along a shock profile as assumed in [24]. ${ }^{1}$ This distinction is unimportant in the small-amplitude case, for which the argument of [24] goes through by continuity assuming only simultaneous symmetrizability at the endstates $\left(u_{-}, v^{*}\left(u_{-}\right)\right){ }^{2}$ however, it becomes significant in the large-amplitude case, for which profiles may feature arbitrarily large excursions from equilibrium.

The difficulty in the analysis of the general case was control of higher-derivative source terms arising in the nonlinear iteration through Taylor expansion of the variable coefficient matrix $A=\left(d f^{t}, d g^{t}\right)^{t}$. Such terms do not arise in the case of discrete kinetic models, for which $A$ is constant, and this made it possible to carry out the entire nonlinear stability analysis using linearized (i.e., Green function) estimates alone. In the general case, we found it necessary to augment these bounds with coupled energy estimates in order to close the iteration, and these estimates, as implemented in [24] used both global symmetrizability and the small-amplitude assumption in important ways. In particular, these assumptions were used to guarantee that the perturbation equations be locally dissipative everywhere along the shock profile, whereas, in the present, large-amplitude case, the perturbation equations are in general dissipative only near plus or minus spatial infinity.

Similar difficulties arose in the closely related study [25] of stability of shock profiles for real viscosity models, initially limiting this analysis also to the smallamplitude, globally symmetrizable case. Recently, however, these obstacles were overcome and the corresponding restrictions removed in [27], by the introduction of a modified energy estimate incorporating "Goodman-type" weighted norms in the style of [12]. As discussed in [39, 40], these quantify the observation that transverse convection relative to the shock profile already yields a complementary type of dissipation near the inner shock layer, by rapidly sweeping signals to plus or minus spatial infinity where they then decay under the effects of the local dissipativivity guaranteed by (A1)-(A2).

In this paper, we show that a similar approach can be applied in the relaxation case to yield a satisfactory nonlinear stability theory applying to large-amplitude profiles of the physically correct class of systems that are simultaneously symmetrizable at equilibrium: indeed, to the considerably more general class of equations that was considered in the linearized analysis of [24].

This brings the analysis of [24] to a satisfying conclusion, putting under a com-

\footnotetext{
${ }^{1}$ This corrects a misstatement in [24], Remark 1.15, where the calculations of [33] were misquoted as asserting global simultaneous symmetrizability.

${ }^{2}$ Specifically, in the notation of Lemma 7.4, [24], the key property $\operatorname{Re}\left(k A-A^{0} Q>0\right.$ persists under perturbation.
} 
mon framework most of the relaxation models commonly studied, and in particular resolving in the negative the question posed in [24] whether there might be a true qualitative difference in behavior between discrete and moment-closure approximations of kinetic models. We point out that our results yield new information even in the case of (non-globally-symmetrizable) discrete kinetic models, since we require significantly less regularity on the data $\left(L^{1} \cap H^{2}\right.$ vs. $\left.W^{3,1} \cap W^{3, \infty}\right)$. Also, the low norm decay rates, $L^{p}$ for $1 \leq p<2$ are new for general models even in the small-amplitude case.

A substantial new difficulty in the relaxation as compared to the real viscosity case is that the hyperbolic characteristic speeds corresponding to eigenvalues of $\left(d f^{t}, d g^{t}\right)^{t}$ are by the subcharacteristic condition (a necessary condition for dissipativity condition (A2); see $[32,24]$ ) necessarily of both positive and negative sign, whereas in the case of real viscosity hyperbolic modes were assumed to be all of one sign, i.e., strictly upwind or strictly downwind. Since weights are chosen to decay exponentially in the direction of propagation, this means that a single scalar weight no longer suffices in the relaxation case, and the introduction of a matrix of distinct diagonal weights leads to off-diagonal error terms that are grow exponentially in both the amplitude of the shock and in an arbitrary constant $C_{*}$ determining the amount of dissipation on the inner layer with respect to the chosen weight.

At first sight, it is hard to see how such an argument could ever close, since good terms of order $C_{*}$ generate bad terms of exponential order in $C_{*}$. Remarkably, the energy estimates can close by a refinement of the hyperbolic compensation argument of $[17,30]$ : namely, the observation (Lemma 2.3 below) that the same "compensating matrix" $K$ used to comple the partial dissipation provided by the semi-parabolic matrix $B$ may be used at the same time to eliminate off-diagonal terms of essentially arbitrary size. The details of this argument may be found in Sections 2-3.

We now describe our results in more detail. Of system (1.2), we assume the following structural properties:

(A1) Symmetrizability of $A(u, v)=\left(d f^{t}, d g^{t}\right)^{t}$ : there exists a $C^{3}$ positive definite matrix-valued function $A^{0}(u, v)$ such that $A^{0} A$ is symmetric.

(A2) Dissipativity at the equilibrium states $\left(u_{ \pm}, v^{*}\left(u_{ \pm}\right)\right)$: for some $\theta>0$,

$$
\operatorname{Re} \sigma\left(i \xi A_{ \pm}+Q_{ \pm}\right) \leq \frac{-\theta|\xi|^{2}}{1+|\xi|^{2}} \quad \forall \xi \in \mathbb{R},
$$

where $A_{ \pm}:=A\left(u_{ \pm}, v^{*}\left(u_{ \pm}\right)\right), Q_{ \pm}:=Q\left(u_{ \pm}, v^{*}\left(u_{ \pm}\right)\right)$, and $Q(u, v):=(0, d q(u, v))^{t}$.

Condition (A1) is connected with well-posedness of (1.2), while (A2) is connected with time-asymptotic stability of equilibrium states $\left(u_{ \pm}, v^{*}\left(u_{ \pm}\right)\right)[17]$. As discussed variously in $[17,35,33,5]$, sufficient conditions for (A2) are either simultaneous symmetrizability, $A^{0} Q$ symmetric, or else weak dissipativity, $Q\left(A^{0}\right)^{-1} \leq 0$ and $\left(Q\left(A^{0}\right)^{-1}\right)_{22}<0,{ }^{3}$ together with genuine coupling:

$$
\text { No eigenvector of } A_{ \pm} \text {lies in the kernel of } Q_{ \pm} \text {. }
$$

\footnotetext{
${ }^{3}$ That is, $\tilde{Q}:=Q\left(A^{0}\right)^{-1}=$ block-diag $\{0, \tilde{q}\}$ with $\tilde{q}<0$, whence we may replace $\tilde{Q}$ with $\operatorname{Re} \tilde{Q}$ and proceed as in the simultaneously symmetrizable case, noting that $\operatorname{ker} A^{0} \operatorname{Re} \tilde{Q}=\operatorname{ker} A^{0} \tilde{Q}=\left(I_{n-r}, 0\right)$, whence (1.3) is preserved. The example $\tilde{q}=\left(\begin{array}{cc}0 & a \\ -a & 1\end{array}\right), a \neq 0$, shows that $\tilde{q}>0$ is necessary, since $\operatorname{Re} \sigma \tilde{q}<0$ but $\operatorname{Re} \tilde{q}=$ block-diag $\{0, \tilde{q}\}$ is only semidefinite.
} 
Regarding the profile (1.1), we assume:

(H0) $f, g, q \in C^{3}$.

(H1) The eigenvalues of $A(x):=\left(d f^{t}, d g^{t}\right)^{t}(\bar{u}, \bar{v})(x)$ are (i) different from $s$ and (ii) of constant multiplicity.

(H2) (i) The eigenvalues $a_{j}^{* \pm}$ of $A_{ \pm}^{*}:=d f^{*}\left(u_{ \pm}\right), f^{*}(u):=f\left(u, v^{*}(u)\right)$, are real and different from $s ;{ }^{4}$ moreover, (ii) when ordered with increasing size, they satisfy the strict Lax characteristic conditions [19]

$$
a_{p-1}^{*-}<0<a_{p}^{*-}, \quad a_{p}^{*+}<0<a_{p+1}^{*+}
$$

for some $1 \leq p \leq n$ (the principal characteristic field of the shock).

(H3) Dynamical stability: the Liu-Majda determinant condition [21, 22, 23, 20]

$$
\Delta:=\operatorname{det}\left(r_{1}^{*-}, \ldots, r_{p-1}^{*-},[u], r_{p+1}^{*+}, r_{n}^{*+}\right) \neq 0
$$

is satisfied, where $r_{j}^{* \pm}$ denote the eigenvectors of $A_{ \pm}^{*}$ associated with $a_{j}^{* \pm}$, and $[u]:=$ $u_{+}-u_{-}$the jump in $u$ across the shock.

(H4) Structural stability: the profile $(\bar{u}, \bar{v})(\cdot)$ is a transverse connection of the associated traveling-wave ODE, in particular, therefore, locally unique up to translation.

(H5) Strong spectral stability: the point spectrum of the linearized operator $\mathcal{L}$ about the wave is contained in $\{\lambda: \operatorname{Re} \lambda<0\} \cup\{0\}$.

Condition (H0) gives the regularity needed both for our analysis here and in order to apply the linearized bounds of [24]. Condition (H1)(i) is a standard assumption $[34,38,24]$ ensuring that the traveling-wave ordinary differential equation (ODE) be of nondegenerate type [24]. It is not clearly necessary, however, and at least for discrete kinetic models it can be relaxed, as we discuss in Section 4; indeed, in that setting it is rather unnatural. Condition (H1)(ii) is a technical assumption that was used in the pointwise Green function analysis of [24]; at the expense of some detail in the pointwise description of linearized behavior, it may be removed altogether [39]. Constant multiplicity holds automatically for discrete kinetic models, but for moment closure models may be difficult to verify. Together, (A1) and (H1)(ii) are equivalent to semisimplicity plus constant multiplicity of $\sigma(A)$. Condition (H2)(i) expresses hyperbolicity and noncharacteristicity of the associated "equilibrium system"

$$
u_{t}+f^{*}(u)_{x}=0
$$

\footnotetext{
${ }^{4}$ This differs from hypothesis $(\mathrm{H} 2)$ of [24], in which the eigenvalues were also required to be distinct. However, it was noted in Remark 1.12 of [24] that this requirement may be dropped when $A_{ \pm}^{*}$ and $Q_{ \pm}$are simultaneously symmetrizable, with essentially no change in either results or notation; the same argument shows that this requirement may likewise be dropped in the general case, at the expense of further complications (specifically, the matrix-valued diffusion waves $\left.e^{\left(x-a_{j}^{* \pm}\right)^{2}\left(4 \pi \beta_{j}^{* \pm}\right.} t\right)^{-1}$ of the remark must be replaced by the fundamental solution of $v_{t}+a_{j}^{* \pm} v_{x}=\beta_{j}^{* \pm} v_{x x}$, where $\beta_{j}^{* \pm}:=$ $l_{j}^{* \pm t} B^{* \pm} r_{j}^{* \pm}$ are no longer necessarily diagonal, and the matrix-valued error-functions appearing in excited term $E$ by its spatial integral; finally, though the precise form of scattering term $S$ in Proposition 1.10 [24] is no longer clear, it is easily verified that it satisfies pointwise bounds yielding the same rates of $L^{q} \rightarrow L^{p}$ decay as needed for subsequent stability arguments).
} 
obtained from (1.2) by formal Chapman-Enskog expansion. at the endstates $u_{-}, u_{+}$ with respect to the corresponding ideal shock $\left(u_{-}, u_{+}\right)$of (1.5). Note that hyperbolicity of the equilibrium system is not required along the profile, thus allowing applications to interesting nonhyperbolic situations as discussed in $[16,1,2]$. Condition (H2)(ii) restricts attention for simplicity to the standard case of a classical, Lax-type shock $\left(u_{-}, u_{+}\right)$of $(1.5)$; the treatment of nonclassical over- and under-compressive shocks we leave for the future. ${ }^{5}$

Conditions (H3)-(H5) are together equivalent to the Evans function condition

$(\mathcal{D})$ The Evans function $D(\cdot)$ associated with $\mathcal{L}$ has precisely one zero on $\{\lambda$ : $\operatorname{Re} \lambda \geq 0\}$ (necessarily at $\lambda=0$ ). ${ }^{6}$

The generalized spectral stability condition $(\mathcal{D})$ was shown in [24] to be necessary and sufficient for linearized stability under assumptions (A1)-(A2) and (H0)-(H2); the main point of this paper is to show that these conditions are sufficient also for nonlinear stability. The conditions (H3) and (H4) correspond to the classical physical notions of dynamical and structural stability (see, e.g. [3]), whereas (H5) encodes heretofor neglected relaxation effects; for further discussion in the closely related viscous case, see [38] and especially [27]. All three of conditions (H3)-(H5) hold automatically in the small-amplitude case; see [21, 22, 23, 34, 24, 28], respectively.

Definition 1.1. For a profile $\bar{U}=(\bar{u}, \bar{v})$ that is (as in the Lax case) unique up to translation, we define NONLINEAR ORBITAL STABILITY as convergence of $U=$ $(u, v)(\cdot, t)$ as $t \rightarrow \infty$ to a translate $\bar{U}(\cdot-\delta(t))$, where $\delta(\cdot)$ is an appropriately chosen function describing shock location, for any solution $U$ of (1.2) with initial data sufficiently close in some norm to the the original profile $\bar{U}$.

Then, the main result of this paper is:

TheOREm 1.2. Let $\bar{U}=(\bar{u}, \bar{v})$ be a profile (1.1) of a relaxation system (1.2), under assumptions (A1)-(A2) and (H0)-(H5). Then, $\bar{U}$ is nonlinearly orbitally stable from $L^{1} \cap H^{2}$ to $L^{p}$, for all $p \geq 2$.

More precisely, for initial perturbations $U_{0}:=\tilde{U}_{0}-\bar{U}$ with $\left|U_{0}\right|_{L^{1} \cap H^{2}}$ sufficiently small, the solution $\tilde{U}=(\tilde{u}, \tilde{v})(x, t)$ of (1.2) with initial data $\tilde{U}_{0}$ satisfies

$$
|\tilde{U}(x, t)-\bar{U}(x-\delta(t))|_{L^{p}} \leq C\left|U_{0}\right|_{L^{1} \cap H^{2}}(1+t)^{-\frac{1}{2}\left(1-\frac{1}{p}\right)}
$$

for all $1 \leq p \leq \infty$, for some $\delta(t)$ satisfying

$$
|\dot{\delta}(t)| \leq C\left|U_{0}\right|_{L^{1} \cap H^{2}}(1+t)^{-\frac{1}{2}} \quad \text { and } \quad|\delta(t)| \leq C\left|U_{0}\right|_{L^{1} \cap H^{2}}
$$

REMARK 1.3. Useful geometric necessary conditions for viscous stability have been obtained in $[36,38,11]$ in the simultaneously symmetrizable case (A3)(ii) using the stability index of $[10,4]$. Strengthened, signed versions $\Delta>0$ (under appropriate normalization) of the dynamical stability condition (1.4), these readily yield examples of spectrally unstable large-amplitude profiles, similarly as in the strictly parabolic case (see, e.g., $[10,7,42,38])$. This shows that the stability conditions assumed in

\footnotetext{
${ }^{5}$ See $[24]$ for a linearized analysis and $[41,38,13]$ for related nonlinear analyses in the viscous or viscous-dispersive case.

${ }^{6}$ For a precise definition of the Evans function, and a proof of the equivalence of $(\mathcal{D})$ and $(\mathrm{H} 3)-$ (H5), see [24], or Appendix A2, [38].
} 
Theorem 1.2 are not vacuous in the large amplitude case. Moreover, as discussed in [38], Section 6.2, the signed version of the Majda condition can serve as a physical selection principle in situations for neither of the classical criteria of structural or dynamical stability suffice. As discussed further in [38, 27], it is an extremely interesting open problem which of the stability conditions (H3) and (H5) is in practice most restrictive.

Similarly, as in the small-amplitude case, Theorem 1.2 is obtained by a nonlinear iteration combining the linearized decay rates of [24] with an appropriate auxiliary energy estimate controlling higher derivatives. Following [24], define the nonlinear perturbation $U=(u, v)$ by

$$
U(x, t):=\tilde{U}(x+\delta(t), t)-\bar{U}(x)
$$

where the "shock location" $\delta$ is to be determined later. Evidently, decay of $U$ is equivalent to nonlinear orbital stability as described in (1.6). Then, the key energy estimate, and the main technical contribution of the paper, is as follows.

Proposition 1.4. Under the hypotheses of Theorem 1.2, let $U_{0} \in H^{2}$, and suppose that, for $0 \leq t \leq T$, both the supremum of $|\dot{\delta}|$ and the $H^{1} \cap W^{1, \infty}$ norm of the solution $U=(u, v)^{\bar{t}}$ of (1.7) remain bounded by a sufficiently small constant $\zeta>0$. Then, for all $0 \leq t \leq T$ and some $\theta>0$,

$$
|U|_{H^{2}}^{2}(t) \leq C e^{-\theta t}\left|U_{0}\right|_{H^{2}}^{2}+C \int_{0}^{t} e^{-\theta(t-s)}\left(|U|_{L^{2}}^{2}+\dot{\delta}^{2}\right)(s) d s .
$$

Inequality (1.8), expressing exponential damping of high frequencies, improves the weaker bound

$$
|U|_{H^{2}}^{2}(t)+\int_{0}^{t}\left|U_{x}\right|_{H^{1}}^{2}(s) d s \leq C\left(\zeta^{2}+\left|U_{0}\right|_{H^{2}}^{2}\right)+C \int_{0}^{t}\left(|U|_{H^{1}}^{2}+\dot{\delta}^{2}\right)(s) d s
$$

stated for the small-amplitude case in [24]. As discussed in [39, 40] in the context of real viscosity systems, both bounds follow from the same string of energy estimates; similar inequalities hold in the real viscosity case.

With estimate (1.9), Theorem 1.2 follows for high norms $L^{p}, 2 \leq p \leq \infty$, by exactly the same argument used in [24] to treat the small-amplitude case; for, the proof of (1.9) was the single place in [24] where the small-amplitude assumption was actually used. See Section 7 of [24], or Section 4, [27] in the real viscosity case. For completeness, we give in Section 4 a simplified version of this argument based on the improved estimate (1.8) of Proposition 1.4, which suffices for low norms $L^{p}, 1 \leq p<2$, as well.

REMARK 1.5. We have here restricted for simplicity to the study of Lax-type shocks. Nonclassical over- and undercompressive shocks may be treated similarly under further restrictions on the initial data; see [14, 29] for analyses in the parabolic (resp. hyperbolic-parabolic) case.

Plan of the paper. In Section 2, we the preliminary lemmas needed in the analysis. In Section 3, we carry out the proof of Proposition 1.4 and in Section 4 the proof of Theorem 1.2. Finally, in Section 5, we discuss the complementary characteristic case. 
2. Preliminaries. As in [27], our starting point consists in the following two lemmas.

LEMMA 2.1. [26] Under assumptions (A1)-(A2), (HO)-(H2), standing wave solutions (1.1) satisfy

$$
\left|(d / d x)^{k} \bar{U}-U_{ \pm}\right| \leq C\left|\bar{U}_{x}\right| \leq C e^{-\theta|x|}, \quad k=0, \ldots, 4,
$$

as $x \rightarrow \pm \infty$, for some $\theta>0, U_{ \pm}=\left(u_{ \pm}, v_{ \pm}\right)=\left(u_{ \pm}, v^{*}\left(u_{ \pm}\right)\right)$.

Proof. Equivalently, the standing-wave equations may be expressed as a nondegenerate ODE with hyperbolic rest points; see the proof of Lemma 1.2, [24].

LemMa 2.2. [30] Let $A$ and $Q$ denote simultaneously symmetrizable matrices and $A^{0}$ their symmetrizer, with $A^{0} Q \leq 0$.

Then, genuine coupling (1.3) is equivalent to one of the following conditions:

(KO) There exists $\theta>0$ such that $\operatorname{Re} \sigma(i \xi A+Q) \leq \frac{-\theta|\xi|^{2}}{1+|\xi|^{2}}$ for all $\xi \in \mathbb{R}$;

(K1) There exists a smooth skew-symmetric matrix-valued function $K\left(A, Q, A^{0}\right)$ such that $\operatorname{Re}\left(A^{0} Q-K A\right)<0$;

(K2) block-diag $L Q R<0$, where $L:=O^{t}\left(A^{0}\right)^{\frac{1}{2}}$ and $R:=L^{-1}=\left(A^{0}\right)^{-\frac{1}{2}} O$ are $m a$ trices of left and right eigenvectors of $A$ block-diagonalizing $L A R$, with $O$ orthonormal. Here, block-diag $M$ denotes the matrix formed from the diagonal blocks of $M$, with each blocks of dimension equal to the multiplicity of corresponding eigenvalues of $L A R$.

Note that strictly dissipativity assumption (A2) correspond to condition (K0) at the asymptotic states $\left(u_{ \pm}, v^{*}\left(u_{ \pm}\right)\right)$with respect to the matrices $A_{ \pm}$and $Q_{ \pm}$.

Proof. These and other useful equivalent formulations are established in [30]. The main implication for our purposes, $(\mathrm{K} 2) \Rightarrow(\mathrm{K} 1)$, follows readily from Lemma 2.3 , below, by first converting to the case of symmetric $A, Q$ by the transformations $\left.\left.A \rightarrow\left(A^{0}\right)^{\frac{1}{2}} A A^{0}\right)^{-\frac{1}{2}}, Q \rightarrow\left(A^{0}\right)^{\frac{1}{2}} Q A^{0}\right)^{-\frac{1}{2}}$, from which the orginal result follows by the fact that $M>0 \Leftrightarrow\left(A^{0}\right)^{\frac{1}{2}} M\left(A^{0}\right)^{\frac{1}{2}}>0$, then converting by an orthonormal change of coordinates to the case that $A$ is diagonal and $Q$ symmetric. Variable multiplicity eigenvalues may be handled by partition of unity/interpolation, noting that $\operatorname{Re}(Q-K A)<0$ persists under perturbation.

Under the assumed symmetry of $L Q R,(\mathrm{~K} 0) \Rightarrow(\mathrm{K} 2)$ follows by Taylor expansion at infinity of the spectrum of the symbol $i \xi A+Q$, from which we may deduce

$$
\operatorname{Re} \sigma(\text { block-diag } L Q R)<0 ;
$$

see, e.g., Appendix B of [24]. That $(\mathrm{K} 1) \Rightarrow(\mathrm{K} 0)$ follows upon rearrangement of energy estimate

$$
\left\langle\left(A^{0}+|\xi|^{2} A^{0}-i \xi K\right) w,\left(\lambda+i \xi A+|\xi|^{2} B\right) w\right\rangle=0 .
$$

Finally, $(\mathrm{K} 2) \Leftrightarrow(1.3)$ is clear.

For our purposes, we shall require the following slight extension of Lemma 2.2, whose proof gives at the same time an explicit description of $K$ of which we shall later make important use. We note that an equivalent version of this result was obtained independently and previously to ours by Humpherys [15]. 
LEMMA 2.3. Let $D$ be diagonal, with real entries appearing with prescribed multiplicity in order of increasing size, and let $Q$ be arbitrary. Then, there exists a smooth skew-symmetric matrix-valued function $K(D, Q)$ such that

$$
\operatorname{Re}(Q-K D)=\text { Re block-diag } Q,
$$

where block-diag $Q$ denotes the block-diagonal part of $Q$, with blocks of dimension equal to the multiplicity of the corresponding eigenvalues of $D$.

Proof. It is straightforward to check that the symmetric matrix $\operatorname{Re} K D=\left(\frac{1}{2}\right)(K D-$ $\left.D^{t} K\right)$ may be prescribed arbitrarily on off-diagonal blocks, by setting $K_{i j}:=\left(a_{i}-\right.$ $\left.a_{j}\right)^{-1} M_{i j}$, where $M_{i j}$ is the desired block, $i \neq j$. Choosing $M=\operatorname{Re} Q$, we obtain $\operatorname{Re}(Q-K D)=$ Re block-diag $(Q)$ as claimed.

We shall also need the following two elementary results.

LEMmA 2.4. Given (A2), there exist block-diagonalizing matrices $L_{ \pm}, R_{ \pm}, L A R_{ \pm}$ blockdiagonal, $L R_{ \pm}=I$, such that

$$
\text { block-diag } L Q R_{ \pm}<0 .
$$

(Note: $A$ and $Q$ are not assumed simultaneously symmetrizable as in Lemma 2.2).

Proof. Again, $\operatorname{Re} \sigma$ (block-diag $\left.\tilde{L} Q \tilde{R}_{ \pm}\right)<0$ follows from (A2) by Taylor expansion at infinity of the spectrum of the symbol $i \xi A_{ \pm}+Q_{ \pm}$, for any block-diagonalizing transformations $\tilde{L}_{ \pm}, \tilde{R}_{ \pm}$; see Appendix B, [24]. By a standard linear-algebraic lemma (see, e.g., Proposition A.9, p. 361 of [31]), block-diag $S^{-1} \tilde{L} Q \tilde{R} S_{ \pm}<0, S_{ \pm}:=$ block-diag $\left\{S_{1}, \ldots, S_{k}\right\}_{ \pm}$for some choice of nonsingular $S_{j}^{ \pm}$. Taking $L_{ \pm}:=S^{-1} \tilde{L}_{ \pm}$, $R_{ \pm}:=\tilde{R} S_{ \pm}$, we are done.

LEMMA 2.5. There is a correspondence between symmetric positive definite symmetrizers $A^{0}, A^{0} A$ symmetric, and diagonalizing transformations $L, R, L A R$ diagonal, given by $A^{0}=L^{*} L$, or equivalently $L=O^{*}\left(A^{0}\right)^{\frac{1}{2}}$, where $O$ is an orthonormal matrix diagonalizing the symmetric matrix $\left(A^{0}\right)^{\frac{1}{2}} A\left(A^{0}\right)^{-\frac{1}{2}}$.

Moreover, the matrix $O$ (or equivalently $L$ ) may be chosen with the same degree of smoothness as $A^{0}$, on any simply connected domain.

Proof. The first assertion follows by direct calculation. The second is clear in the strictly hyperbolic case, for which the correspondence is also one-to-one; in the general (constant-multiplicity) case, it follows by a standard lemma of Kato [18].

REMARK 2.6. Lemma 2.5 hints at the strategy we shall follow in carrying out energy estimates, which is to "effectively diagonalize" by the use of a symmetrizer. That is, rather than working with $L A R$ as we should like, we work with $A^{0} A=$ $L^{*} L A=L^{*}(L A R) L$, thereby avoiding the problem that there may exist a nonlinear change of coordinates with Jacobian $L$. Conjugation by $L$ of course does not affect the energy estimates.

Finally, for convenience of the reader, we recall the standard relations

$$
\left\langle W, S W_{x}\right\rangle=-\frac{1}{2}\left\langle W, S_{x} W\right\rangle
$$

and

$$
\frac{1}{2}\left\langle W_{x}, K W\right\rangle_{t}=\left\langle W_{x}, K W_{t}\right\rangle+\frac{1}{2}\left\langle W_{x}, K_{t} W\right\rangle+\frac{1}{2}\left\langle W, K_{x} W_{t}\right\rangle,
$$

valid, respectively, for symmetric $S$ and skew-symmetric $K$. 
3. Energy estimates.. In this section, we carry out the main work of the paper, establishing Proposition 1.4.

Perturbation equation. Define the nonlinear perturbation $U(x, t):=\tilde{U}(x+\delta(t), t)-$ $\bar{U}(x)$ as in (1.7) where $\delta(t)$ (estimating shock location) is to be determined later; for definiteness, fix $\delta(0)=0$. Substituting (1.7) into (1.2), we obtain

$$
\tilde{U}_{t}+\tilde{A} \tilde{U}_{x}-\left(\begin{array}{c}
0 \\
q
\end{array}\right)(\tilde{U})=\dot{\delta} \tilde{U}_{x}
$$

where $\tilde{A}:=(d f, d g)^{t}(\tilde{u}, \tilde{v})$, and thereby

$$
(\tilde{U}-\bar{U})_{t}+\left(\tilde{A} \tilde{U}_{x}-\bar{A} \bar{U}_{x}\right)-\left(\left(\begin{array}{c}
0 \\
q
\end{array}\right)(\tilde{U})-\left(\begin{array}{c}
0 \\
q
\end{array}\right)(\bar{U})\right)=\dot{\delta}(t) \tilde{U}_{x}
$$

where $\tilde{U}$ now denotes $\tilde{U}(x+\delta(t), t)$ and $\bar{U}$ denotes $\bar{U}(x)$.

Expanding $\left(\tilde{A} \tilde{U}_{x}-\bar{A} \bar{U}_{x}\right)$ using the quadratic Leibnitz relation

$$
\tilde{A} \tilde{U}_{x}-\bar{A} \bar{U}_{x}=\tilde{A}\left(\tilde{U}_{x}-\bar{U}_{x}\right)+(\tilde{A}-\bar{A}) \bar{U}_{x}
$$

and Taylor expanding $\left(\left(\begin{array}{l}0 \\ q\end{array}\right)(\tilde{U})-\left(\begin{array}{l}0 \\ q\end{array}\right)(\bar{U})\right)$ about $\tilde{U}$, we obtain the basic nonlinear perturbation equation

$$
U_{t}-\tilde{A} U_{x}-\tilde{Q} U=M_{1}(U) \bar{U}_{x}+\left(0, I_{r}\right)^{t} M_{2}(U)+\dot{\delta}(t)\left(\bar{U}_{x}+U_{x}\right),
$$

where $\tilde{Q}:=(0, d q)^{t}(\tilde{u}, \tilde{v})$ and

$$
\begin{aligned}
& M_{1}(U)=\mathcal{O}(|U|):=\tilde{A}(x, t)-\bar{A}(x) \\
& M_{2}(U)=\mathcal{O}\left(|U|^{2}\right):=\left(\begin{array}{l}
0 \\
q
\end{array}\right)(\tilde{U})-\left(\begin{array}{l}
0 \\
q
\end{array}\right)(\bar{U})-\tilde{Q}(\tilde{U}-\bar{U}) .
\end{aligned}
$$

Weighting matrix. Let $\tilde{A}^{0}:=A^{0}(\tilde{U})$ denote the symmetrizer of $\tilde{A}$ guaranteed by (A1), and factor $\tilde{A}^{0} \tilde{A}=\left(\tilde{A}^{0}\right)^{\frac{1}{2}} \tilde{O} \tilde{D} \tilde{O}^{t}\left(\tilde{A}^{0}\right)^{\frac{1}{2}}$, or, equivalently,

$$
\tilde{A}=\left(\tilde{A}^{0}\right)^{-\frac{1}{2}} \tilde{O} \tilde{D} \tilde{O}^{t}\left(\tilde{A}^{0}\right)^{\frac{1}{2}},
$$

where $\tilde{O}$ is orthogonal, $\tilde{O}^{t}=\tilde{O}^{-1}$, and $C^{3}$ as a function of $(u, v)$ (see Lemma 2.5) and $\tilde{D}=$ block-diag $\left\{\tilde{a}_{1}, \ldots, \tilde{a}_{l}\right\}$, where $\tilde{a}_{j}$ denote the eigenvalues of $\tilde{A}$, indexed in increasing order

$$
\tilde{a}_{1} \leq \cdots \leq \tilde{a}_{k}<0<\tilde{a}_{k+1} \leq \cdots \leq \tilde{a}_{l}
$$

Define the "Goodman-type" [12] weighting matrix $\alpha(x):=\operatorname{block-diag}\left\{\alpha_{1}, \ldots, \alpha_{l}\right\}$, where $\alpha_{j}>0$ are defined by ODE

$$
\alpha_{x}=C_{*} \operatorname{sgn} a_{j}\left|\bar{U}_{x}\right| \alpha, \quad \alpha(0)=1,
$$

$C_{*}>0$ a sufficiently large constant to be determined later. This definition, together with $\left|a_{j}\right|>0,(\mathrm{H} 2)(\mathrm{i})$, gives the key inequality

$$
\alpha_{x} \bar{D} \geq \theta_{1} C_{*}\left|\bar{U}_{x}\right| \alpha,
$$


where $\bar{D}=$ block-diag $\left\{\bar{a}_{1}, \ldots, \bar{a}_{l}\right\}$ and $\bar{a}_{j}$ are the eigenvalues of $\bar{A}$.

Setting

$$
\tilde{A}_{\alpha}^{0}:=\left(\tilde{A}^{0}\right)^{\frac{1}{2}} \tilde{O} \alpha \tilde{O}^{t}\left(\tilde{A}^{0}\right)^{\frac{1}{2}}
$$

we have by factorization (3.2) that

$$
\tilde{A}_{\alpha}^{0} \tilde{A}=\left[\left(\tilde{A}^{0}\right)^{\frac{1}{2}} \tilde{O} \alpha \tilde{O}^{t}\left(\tilde{A}^{0}\right)^{\frac{1}{2}}\right] \tilde{A}=\left(\tilde{A}^{0}\right)^{\frac{1}{2}} \tilde{O}(\alpha \tilde{D}) \tilde{O}^{t}\left(\tilde{A}^{0}\right)^{\frac{1}{2}} .
$$

Hence, $\tilde{A}_{\alpha}^{0} \tilde{A}$ is symmetric and the symmetric positive definite matrix $\tilde{A}_{\alpha}^{0}$ is also a viable symmetrizer for $\tilde{A}$.

Moreover, setting $L:=\tilde{O}^{t}\left(\tilde{A}^{0}\right)^{\frac{1}{2}}$ and $R:=\left(\tilde{A}^{0}\right)^{-\frac{1}{2}} \tilde{O}$, by Lemma 2.5 and constant multiplicity of eigenvalues of $A$, we have the freedom to smoothly $\left(C^{3}\right)$ redefine $L$ and $R$ so that they take on prescribed values at $x \rightarrow \pm \infty$. Thus, by Lemma 2.4, we may assume without loss of generality that

$$
\text { Re block-diag }\left(\alpha \tilde{O}^{t}\left(\tilde{A}^{0}\right)^{\frac{1}{2}} \tilde{Q}\left(\tilde{A}^{0}\right)^{-\frac{1}{2}} \tilde{O}\right)_{ \pm} \leq-\theta\left(C_{*}\right)<0,
$$

and thereby, appealing to $(2.1), k=0$,

$$
\text { Re block-diag }\left(\alpha \tilde{O}^{t}\left(\tilde{A}^{0}\right)^{\frac{1}{2}} \tilde{Q}\left(\tilde{A}^{0}\right)^{-\frac{1}{2}} \tilde{O}\right) \leq-\theta\left(C_{*}\right)+C\left(C_{*}\right) \zeta+C\left|\bar{U}_{x}\right| \alpha,
$$

where

$$
\tilde{A}_{\alpha}^{0} \tilde{Q}=\left(\tilde{A}^{0}\right)^{\frac{1}{2}} \tilde{O}\left[\alpha \tilde{O}^{t}\left(\tilde{A}^{0}\right)^{\frac{1}{2}} \tilde{Q}\left(\tilde{A}^{0}\right)^{-\frac{1}{2}} \tilde{O}\right] \tilde{O}^{t}\left(\tilde{A}^{0}\right)^{\frac{1}{2}},
$$

and the exponent $\theta$ now refers to the minimum of the constants used elsewhere in the argument and that appearing in (2.1). The constants $\theta\left(C_{*}\right)$ and $C\left(C_{*}\right)$ measure the conditioning of matrices $\alpha$ and in fact decay (resp. grow) exponentially with respect to $C_{*}$; however, this is unimportant for our argument.

Define

$$
K_{1}:=K\left(\tilde{D}, \alpha \tilde{O}^{t}\left(\tilde{A}^{0}\right)^{\frac{1}{2}} \tilde{Q}\left(\tilde{A}^{0}\right)^{-\frac{1}{2}} \tilde{O}+N\right),
$$

where $K(\cdot)$ is as in Lemma 2.3, and $N$ is an arbitrary matrix with $|N|_{C_{x, t}^{1}} \leq C\left(C_{*}\right)$ and vanishing on diagonal blocks, to be determined later. Moreover, let $\tilde{K}_{\alpha}$ be the skew-symmetric matrix obtained from $K_{1}$ after conjugation by $\left(\tilde{A}^{0}\right)^{\frac{1}{2}} \tilde{O}$, i.e.

$$
\tilde{K}_{\alpha}:=\left(\tilde{A}^{0}\right)^{\frac{1}{2}} \tilde{O} K_{1} \tilde{O}^{t}\left(\tilde{A}^{0}\right)^{\frac{1}{2}} .
$$

For later use, note that, through smooth dependence on $\tilde{U}=\bar{U}+U$ and $N$,

$$
\left|\tilde{K}_{\alpha, x}\right|,\left|\tilde{K}_{\alpha, t}\right| \leq C\left(C_{*}\right) .
$$

We have, therefore,

$$
\begin{aligned}
& \operatorname{Re}\left(-\tilde{K}_{\alpha} \tilde{A}+\tilde{A}_{\alpha}^{0} \tilde{Q}+\left(\tilde{A}^{0}\right)^{\frac{1}{2}} \tilde{O} N \tilde{O}^{t}\left(\tilde{A}^{0}\right)^{\frac{1}{2}}\right) \\
& \quad=\operatorname{Re}\left(\tilde{A}^{0}\right)^{\frac{1}{2}} \tilde{O}\left(-K_{1} \tilde{D}+\alpha \tilde{O}^{t}\left(\tilde{A}^{0}\right)^{\frac{1}{2}} \tilde{Q}\left(\tilde{A}^{0}\right)^{-\frac{1}{2}} \tilde{O}+N\right) \tilde{O}^{t}\left(\tilde{A}^{0}\right)^{\frac{1}{2}} \\
& \quad=\operatorname{Re}\left(\tilde{A}^{0}\right)^{\frac{1}{2}} \tilde{O} \text { block-diag }\left(\alpha \tilde{O}^{t}\left(\tilde{A}^{0}\right)^{\frac{1}{2}} \tilde{Q}\left(\tilde{A}^{0}\right)^{-\frac{1}{2}} \tilde{O}\right) \tilde{O}^{t}\left(\tilde{A}^{0}\right)^{\frac{1}{2}} \\
& \quad \leq-\theta\left(C_{*}\right)+C\left(C_{*}\right) \zeta+C\left|\bar{U}_{x}\right| \tilde{A}_{\alpha}^{0}
\end{aligned}
$$


by (3.6) together with Lemma 2.3. By (3.3), we have also

$$
\begin{array}{r}
\left(\tilde{A}^{0}\right)^{\frac{1}{2}} \tilde{O}\left(\alpha_{x} \tilde{D}\right) \tilde{O}^{t}\left(\tilde{A}^{0}\right)^{\frac{1}{2}} \geq\left(\tilde{A}^{0}\right)^{\frac{1}{2}} \tilde{O}\left(\alpha_{x} \bar{D}\right) \tilde{O}^{t}\left(\tilde{A}^{0}\right)^{\frac{1}{2}}-C\left(C_{*}\right) \zeta \\
\geq \theta C_{*}\left|\bar{U}_{x}\right| \tilde{A}_{\alpha}^{0}-C\left(C_{*}\right) \zeta
\end{array}
$$

for possibly still smaller $\theta>0$ ( $\zeta$ as defined in the statement of Proposition 1.4).

Friedrichs-type estimate. As in [24], we first perform a standard "Friedrichs-type" estimate for symmetric hyperbolic systems (see $[8,9]$ ), now incorporating the weight $\alpha$.

Differentiating (3.1) twice with respect to $x$, we obtain

$$
U_{x x t}-\left(\tilde{A} U_{x}\right)_{x x}-(\tilde{Q} U)_{x x}=\left(M(U) \bar{U}_{x}\right)_{x x}+\left(0, I_{r}\right)^{t} M_{2}(U)_{x x}+\dot{\delta}(t)\left(\bar{U}_{x x x}+U_{x x x}\right) .
$$

Taking the $L^{2}$ inner product $\tilde{A}_{\alpha}^{0} U_{x x}$ against $U_{x x t}$, we get

$$
\frac{1}{2}\left\langle\tilde{A}_{\alpha}^{0} U_{x x}, U_{x x}\right\rangle_{t}=\left\langle\tilde{A}_{\alpha}^{0} U_{x x}, U_{x x t}\right\rangle+\frac{1}{2}\left\langle\left(\tilde{A}_{\alpha}^{0}\right)_{t} U_{x x}, U_{x x}\right\rangle
$$

where $\tilde{A}_{\alpha}^{0}$ is defined in (3.4).

The second term can be easily bounded: indeed, using (3.1),

$\left|\left(\tilde{A}_{\alpha}^{0}\right)_{t}\right|_{L^{\infty}}=\left|\frac{d A_{\alpha}^{0}}{d \tilde{U}}\right|\left|\tilde{U}_{t}\right|=\left|\frac{d A_{\alpha}^{0}}{d \tilde{U}}\right|\left|U_{t}\right| \leq C\left(C_{*}\right)\left[\left|U_{W^{1, \infty}}+\right| \dot{\delta}(t) \mid\left(\left|\bar{U}_{x}\right|_{L^{\infty}}+\left|U_{x}\right|_{L^{\infty}}\right)\right]$,

hence

$$
\frac{1}{2}\left\langle\left(\tilde{A}_{\alpha}^{0}\right)_{t} U_{x x}, U_{x x}\right\rangle \leq C\left(C_{*}\right) \zeta\left|U_{x x}\right|_{L^{2}}^{2}
$$

( $\zeta$ as defined in the statement of Proposition 1.4).

Let us consider the first term on the righthand side of (3.11)

$$
\begin{array}{r}
\left\langle\tilde{A}_{\alpha}^{0} U_{x x}, U_{x x t}\right\rangle=\left\langle\tilde{A}_{\alpha}^{0} U_{x x},\left(\tilde{A} U_{x}\right)_{x x}\right\rangle+\left\langle\tilde{A}_{\alpha}^{0} U_{x x},(\tilde{Q} U)_{x x}\right\rangle \\
+\left\langle\tilde{A}_{\alpha}^{0} U_{x x},\left(M_{1}(U) \bar{U}_{x}\right)_{x x}\right\rangle \\
+\left\langle\tilde{A}_{\alpha}^{0} U_{x x},\left(0, I_{r}\right)^{t} M_{2}(U)_{x x}\right\rangle \\
+\dot{\delta}(t)\left\langle\tilde{A}_{\alpha}^{0} U_{x x}, \bar{U}_{x x x}+U_{x x x}\right\rangle
\end{array}
$$

Differentiating the first of the terms in (3.13), we get

$$
\left\langle\tilde{A}_{\alpha}^{0} U_{x x},\left(\tilde{A} U_{x}\right)_{x x}\right\rangle=\left\langle\tilde{A}_{\alpha}^{0} U_{x x}, \tilde{A}_{x x} U_{x}\right\rangle+2\left\langle\tilde{A}_{\alpha}^{0} U_{x x}, \tilde{A}_{x} U_{x x}\right\rangle+\left\langle\tilde{A}_{\alpha}^{0} U_{x x}, \tilde{A} U_{x x x}\right\rangle .
$$

Since

$$
\begin{gathered}
\tilde{A}_{x}=\frac{d \tilde{A}}{d \tilde{U}}\left(\bar{U}_{x}+U_{x}\right) \\
\tilde{A}_{x x}=\frac{d^{2} \tilde{A}}{d \tilde{U}^{2}}\left(\bar{U}_{x} \bar{U}_{x}+\bar{U}_{x} U_{x}+U_{x} \bar{U}_{x}+U_{x} U_{x}\right)+\frac{d \tilde{A}}{d \tilde{U}}\left(\bar{U}_{x x}+U_{x x}\right),
\end{gathered}
$$


the first two terms of (3.14) are bounded by

$$
\begin{aligned}
\left\langle\tilde{A}_{\alpha}^{0} U_{x x}, \tilde{A}_{x x} U_{x}\right\rangle+ & 2\left\langle\tilde{A}_{\alpha}^{0} U_{x x}, \tilde{A}_{x} U_{x x}\right\rangle \leq C\left(C_{*}\right)(\zeta+\bar{\zeta})\left|U_{x x}\right|_{L^{2}}^{2} \\
& +C\left(C_{*}, \bar{\zeta}\right)\left|U_{x}\right|_{L^{2}}^{2}+\left\langle\tilde{A}_{\alpha}^{0} U_{x x}, \frac{d \tilde{A}}{d \tilde{U}} \bar{U}_{x} U_{x x}\right\rangle ;
\end{aligned}
$$

here, we have used Young's inequality to bound $\left\langle U_{x}, U_{x x}\right\rangle$ with $\bar{\zeta}\left|U_{x x}\right|_{L^{2}}^{2}+C\left|U_{x}\right|_{L^{2}}^{2}$ with $\bar{\zeta}>0$ chosen such that $\zeta \ll \bar{\zeta} \ll 1$.

Using the symmetry of $\tilde{A}_{\alpha}^{0}$ and $(2.2)$ with $S=\tilde{A}_{\alpha}^{0} \tilde{A}$, we find that the last term of (3.14) takes the form

$$
\left\langle\tilde{A}_{\alpha}^{0} U_{x x}, \tilde{A} U_{x x x}\right\rangle=\left\langle U_{x x}, \tilde{A}_{\alpha}^{0} \tilde{A} U_{x x x}\right\rangle=-\frac{1}{2}\left\langle U_{x x},\left(\tilde{A}_{\alpha}^{0} \tilde{A}\right)_{x} U_{x x}\right\rangle .
$$

Recalling (3.5), we have

$$
\left(\tilde{A}_{\alpha}^{0} \tilde{A}\right)_{x}=\frac{d\left(\tilde{A}_{\alpha}^{0} \tilde{A}\right)}{d \tilde{U}}\left(\bar{U}_{x}+U_{x}\right)+\left(\tilde{A}^{0}\right)^{\frac{1}{2}} \tilde{O}\left(\alpha_{x} \tilde{D}\right) \tilde{O}^{t}\left(\tilde{A}^{0}\right)^{\frac{1}{2}}
$$

where, with slight abuse of notation,

$$
\frac{d\left(\tilde{A}_{\alpha}^{0} \tilde{A}\right)}{d \tilde{U}} W=\frac{d\left(\left(\tilde{A}^{0}\right)^{\frac{1}{2}} \tilde{O}\right)}{d \tilde{U}} W \alpha \tilde{D} \tilde{O}^{t}\left(\tilde{A}^{0}\right)^{\frac{1}{2}}+\left(\tilde{A}^{0}\right)^{\frac{1}{2}} \tilde{O} \alpha \frac{d\left(\tilde{D} \tilde{O}^{t}\left(\tilde{A}^{0}\right)^{\frac{1}{2}}\right)}{d \tilde{U}} W .
$$

Hence, we get

$$
\begin{array}{r}
\left\langle\tilde{A}_{\alpha}^{0} U_{x x}, \tilde{A} U_{x x x}\right\rangle \leq-\frac{1}{2}\left\langle U_{x x},\left(\tilde{A}^{0}\right)^{\frac{1}{2}} \tilde{O}\left(\alpha_{x} \tilde{D}\right) \tilde{O}^{t}\left(\tilde{A}^{0}\right)^{\frac{1}{2}} U_{x x}\right\rangle \\
+C\left(C_{*}\right) \zeta\left|U_{x x}\right|_{L^{2}}^{2}-\frac{1}{2}\left\langle U_{x x}, \frac{d\left(\tilde{A}_{\alpha}^{0} \tilde{A}\right)}{d \tilde{U}} \bar{U}_{x} U_{x x}\right\rangle
\end{array}
$$

Summarizing, the first term on the righthand side of (3.13) can be estimated by

$$
\begin{array}{r}
\left\langle\tilde{A}_{\alpha}^{0} U_{x x},\left(\tilde{A} U_{x}\right)_{x x}\right\rangle \leq C\left(C_{*}\right)(\zeta+\bar{\zeta})\left|U_{x x}\right|_{L^{2}}^{2}+C\left(C_{*}, \bar{\zeta}\right)\left|U_{x}\right|_{L^{2}}^{2} \\
+\left\langle\tilde{A}_{\alpha}^{0} U_{x x}, \frac{d \tilde{A}}{d \tilde{U}} \bar{U}_{x} U_{x x}\right\rangle-\frac{1}{2}\left\langle U_{x x},\left(\tilde{A}^{0}\right)^{\frac{1}{2}} \tilde{O}\left(\alpha_{x} \tilde{D}\right) \tilde{O}^{t}\left(\tilde{A}^{0}\right)^{\frac{1}{2}} U_{x x}\right\rangle \\
-\frac{1}{2}\left\langle U_{x x}, \frac{d\left(\tilde{A}_{\alpha}^{0} \tilde{A}\right)}{d \tilde{U}} \bar{U}_{x} U_{x x}\right\rangle .
\end{array}
$$

The second term in (3.13) can be dealt with similarly: since

$\tilde{Q}_{x}=\frac{d \tilde{Q}}{d \tilde{U}}\left(\bar{U}_{x}+U_{x}\right), \quad \tilde{Q}_{x x}=\frac{d^{2} \tilde{Q}}{d \tilde{U}^{2}}\left(\bar{U}_{x} \bar{U}_{x}+\bar{U}_{x} U_{x}+U_{x} \bar{U}_{x}+U_{x} U_{x}\right)+\frac{d \tilde{Q}}{d \tilde{U}}\left(\bar{U}_{x x}+U_{x x}\right)$,

we have

$$
\begin{array}{r}
\left\langle\tilde{A}_{\alpha}^{0} U_{x x},(\tilde{Q} U)_{x x}\right\rangle=\left\langle\tilde{A}_{\alpha}^{0} U_{x x}, \tilde{Q}_{x x} U\right\rangle+2\left\langle\tilde{A}_{\alpha}^{0} U_{x x}, \tilde{Q}_{x} U_{x}\right\rangle+\left\langle\tilde{A}_{\alpha}^{0} U_{x x}, \tilde{Q} U_{x x}\right\rangle \\
\leq C\left(C_{*}\right)(\zeta+\bar{\zeta})\left|U_{x x}\right|_{L^{2}}^{2}+C\left(C_{*}, \bar{\zeta}\right)|U|_{H^{1}}^{2}+\left\langle\tilde{A}_{\alpha}^{0} U_{x x}, \tilde{Q} U_{x x}\right\rangle
\end{array}
$$


with $\bar{\zeta}$ as in the previous case.

The third term in (3.13) can be estimated by

$$
\begin{array}{r}
\left\langle\tilde{A}_{\alpha}^{0} U_{x x},\left(M_{1}(U) \bar{U}_{x}\right)_{x x}\right\rangle \leq C\left(C_{*}\right) \bar{\zeta}\left|U_{x x}\right|_{L^{2}}^{2}+C\left(C_{*}, \bar{\zeta}\right)|U|_{H^{1}}^{2} \\
+\left\langle\tilde{A}_{\alpha}^{0} U_{x x}, \frac{d M_{1}}{d U} U_{x x} \bar{U}_{x}\right\rangle
\end{array}
$$

The fourth term in (3.13) is easier: since

$$
M_{2}(U)_{x x}=\frac{d^{2} M_{2}}{d U^{2}} U_{x} U_{x}+\frac{d M_{2}}{d U} U_{x x},
$$

we have (recall that $M_{2}(U)=\mathcal{O}\left(|U|^{2}\right)$ )

$$
\left\langle\tilde{A}_{\alpha}^{0} U_{x x},\left(0, I_{r}\right)^{t} M_{2}(U)_{x x}\right\rangle \leq C\left(C_{*}\right) \bar{\zeta}\left|U_{x x}\right|_{L^{2}}^{2}+C\left(C_{*}, \bar{\zeta}\right)\left|U_{x}\right|_{L^{2}}^{2} .
$$

Finally, the last term in (3.13): $\dot{\delta}(t)\left\langle\tilde{A}_{\alpha}^{0} U_{x x}, \bar{U}_{x x x}+U_{x x x}\right\rangle$. Since

$$
\dot{\delta}(t)\left\langle\tilde{A}_{\alpha}^{0} U_{x x}, \bar{U}_{x x x}\right\rangle \leq C\left(C_{*}\right)|\dot{\delta}(t)|\left|U_{x x}\right|_{L^{2}} \leq C\left(C_{*}\right) \bar{\zeta}\left|U_{x x}\right|_{L^{2}}^{2}+C\left(C_{*}, \bar{\zeta}\right)|\dot{\delta}(t)|^{2}
$$

and, using (2.2) with $S=\tilde{A}_{\alpha}^{0}$,

$$
\dot{\delta}(t)\left\langle\tilde{A}_{\alpha}^{0} U_{x x}, U_{x x x}\right\rangle=-\frac{1}{2} \dot{\delta}(t)\left\langle U_{x x},\left(\tilde{A}_{\alpha}^{0}\right)_{x} U_{x x}\right\rangle \leq C\left(C_{*}\right) \zeta\left|U_{x x}\right|_{L^{2}}^{2},
$$

we have

$$
\dot{\delta}(t)\left\langle\tilde{A}_{\alpha}^{0} U_{x x}, \bar{U}_{x x x}+U_{x x x}\right\rangle \leq C\left(C_{*}\right) \zeta\left|U_{x x}\right|_{L^{2}}^{2}+C\left(C_{*}\right)|\dot{\delta}(t)| .
$$

Collecting (3.12), (3.19), (3.20), (3.21), (3.22) and (3.23), we get

$$
\begin{array}{r}
\frac{1}{2}\left\langle\tilde{A}_{\alpha}^{0} U_{x x}, U_{x x}\right\rangle_{t} \leq-\frac{1}{2}\left\langle U_{x x},\left(\tilde{A}^{0}\right)^{\frac{1}{2}} \tilde{O}\left(\alpha_{x} \tilde{D}\right) \tilde{O}^{t}\left(\tilde{A}^{0}\right)^{\frac{1}{2}} U_{x x}\right\rangle+\left\langle U_{x x}, \tilde{A}_{\alpha}^{0} \tilde{Q} U_{x x}\right\rangle \\
-\frac{1}{2}\left\langle U_{x x}, \frac{d\left(\tilde{A}_{\alpha}^{0} \tilde{A}\right)}{d \tilde{U}} \bar{U}_{x} U_{x x}\right\rangle+\left\langle U_{x x}, \tilde{A}_{\alpha}^{0}\left(\frac{d \tilde{A}}{d \tilde{U}} \bar{U}_{x} U_{x x}+\frac{d M_{1}}{d U} U_{x x} \bar{U}_{x}\right)\right\rangle \\
+C\left(C_{*}\right)(\zeta+\bar{\zeta})\left|U_{x x}\right|_{L^{2}}^{2}+C\left(C_{*}, \bar{\zeta}\right)\left(|U|_{H^{1}}^{2}+|\dot{\delta}(t)|^{2}\right)
\end{array}
$$

Let us consider the term in (3.24) containing $\frac{d\left(\tilde{A}_{\alpha}^{0} \tilde{A}\right)}{d \tilde{U}}$. By (3.17),

$$
\begin{array}{r}
\left\langle U_{x x}, \frac{d\left(\tilde{A}_{\alpha}^{0} \tilde{A}\right)}{d \tilde{U}} \bar{U}_{x} U_{x x}\right\rangle=\left\langle U_{x x}, \frac{d\left(\left(\tilde{A}^{0}\right)^{\frac{1}{2}} \tilde{O}\right)}{d \tilde{U}} \bar{U}_{x} \alpha \tilde{D} \tilde{O}^{t}\left(\tilde{A}^{0}\right)^{\frac{1}{2}} U_{x x}\right\rangle \\
+\left\langle U_{x x},\left(\tilde{A}^{0}\right)^{\frac{1}{2}} \tilde{O} \alpha \frac{d\left(\tilde{D} \tilde{O}^{t}\left(\tilde{A}^{0}\right)^{\frac{1}{2}}\right)}{d \tilde{U}} \bar{U}_{x} U_{x x}\right\rangle \\
=\left\langle U_{x x},\left(\tilde{A}^{0}\right)^{\frac{1}{2}} \tilde{O} P_{1} \alpha \tilde{O}^{t}\left(\tilde{A}^{0}\right)^{\frac{1}{2}} U_{x x}\right\rangle+\left\langle U_{x x},\left(\tilde{A}^{0}\right)^{\frac{1}{2}} \tilde{O} \alpha P_{2} \tilde{O}^{t}\left(\tilde{A}^{0}\right)^{\frac{1}{2}} U_{x x}\right\rangle,
\end{array}
$$

where

$$
P_{1}=\tilde{O}^{t}\left(\tilde{A}^{0}\right)^{-\frac{1}{2}} \frac{d\left(\left(\tilde{A}^{0}\right)^{\frac{1}{2}} \tilde{O}\right)}{d \tilde{U}} \bar{U}_{x} \tilde{D} \quad \text { and } \quad P_{2}=\frac{d\left(\tilde{D} \tilde{O}^{t}\left(\tilde{A}^{0}\right)^{\frac{1}{2}}\right)}{d \tilde{U}} \bar{U}_{x}\left(\tilde{A}^{0}\right)^{-\frac{1}{2}} \tilde{O}
$$


Similarly,

$$
\left\langle U_{x x}, \tilde{A}_{\alpha}^{0}\left(\frac{d \tilde{A}}{d \tilde{U}} \bar{U}_{x} U_{x x}+\frac{d M_{1}}{d U} U_{x x} \bar{U}_{x}\right)\right\rangle=\left\langle U_{x x},\left(\tilde{A}^{0}\right)^{\frac{1}{2}} \tilde{O} \alpha P_{3} \tilde{O}^{t}\left(\tilde{A}^{0}\right)^{\frac{1}{2}} U_{x x}\right\rangle,
$$

where

$$
P_{3}=\tilde{O}^{t}\left(\tilde{A}^{0}\right)^{\frac{1}{2}}\left(\frac{d \tilde{A}}{d \tilde{U}}+\frac{d M_{1}}{d U}\right) \bar{U}_{x}
$$

The terms

$$
-\frac{1}{2}\left\langle U_{x x},\left(\tilde{A}^{0}\right)^{\frac{1}{2}} \tilde{O} P_{1} \alpha \tilde{O}^{t}\left(\tilde{A}^{0}\right)^{\frac{1}{2}} U_{x x}\right\rangle+\left\langle U_{x x},\left(\tilde{A}^{0}\right)^{\frac{1}{2}} \tilde{O} \alpha\left(P_{3}-\frac{1}{2} P_{2}\right) \tilde{O}^{t}\left(\tilde{A}^{0}\right)^{\frac{1}{2}} U_{x x}\right\rangle
$$

can be rewritten as the sum of two terms, one taking in account "off-block-diagonal" parts (meaning off-block-diagonal after conjugation by $\left.\left(\tilde{A}^{0}\right)^{\frac{1}{2}} \tilde{O}\right)$ and the other taking in account "block-diagonal" parts. We denote the first one as

$$
\left\langle U_{x x},\left(\tilde{A}^{0}\right)^{\frac{1}{2}} \tilde{O} N \tilde{O}^{t}\left(\tilde{A}^{0}\right)^{\frac{1}{2}} U_{x x}\right\rangle,
$$

where $|N|_{C^{1}(x, t} \leq C\left(C_{*}\right)$ indeed holds, since $N$ by definition is of form $J\left(\tilde{U}, \alpha, \bar{U}_{x}\right)$ with $J(\cdot)$ smooth, and $|\tilde{U}|_{C^{1}(x, t)} \leq C \zeta,|\alpha|_{C^{1}(x, t)} \leq C_{*} \sup |\alpha| \leq C\left(C_{*}\right)$. The "blockdiagonal" parts (meaning block-diagonal after conjugation by $\left.\left(\tilde{A}^{0}\right)^{\frac{1}{2}} \tilde{O}\right)$ of these error terms may be estimated by $C\left\langle U_{x x},\left|\bar{U}_{x}\right| \tilde{A}_{\alpha}^{0} U_{x x}\right\rangle$ for some constant $C>0$ (independent on $\left.\alpha, C_{*}\right)$.

Hence, we get the final form of the Friedrichs-type estimate:

$$
\begin{aligned}
\frac{1}{2}\left\langle\tilde{A}_{\alpha}^{0} U_{x x}, U_{x x}\right\rangle_{t} \leq- & \frac{1}{2}\left\langle U_{x x},\left(\tilde{A}^{0}\right)^{\frac{1}{2}} \tilde{O}\left(\alpha_{x} \tilde{D}\right) \tilde{O}^{t}\left(\tilde{A}^{0}\right)^{\frac{1}{2}} U_{x x}\right\rangle+\left\langle U_{x x}, \tilde{A}_{\alpha}^{0} \tilde{Q} U_{x x}\right\rangle \\
& +\left\langle U_{x x},\left(\tilde{A}^{0}\right)^{\frac{1}{2}} \tilde{O} N \tilde{O}^{t}\left(\tilde{A}^{0}\right)^{\frac{1}{2}} U_{x x}\right\rangle+C\left\langle U_{x x},\left|\bar{U}_{x}\right| \tilde{A}_{\alpha}^{0} U_{x x}\right\rangle \\
& +C\left(C_{*}\right)(\zeta+\bar{\zeta})\left|U_{x x}\right|_{L^{2}}^{2}+C\left(C_{*}, \bar{\zeta}\right)\left(|U|_{H^{1}}^{2}+|\dot{\delta}(t)|^{2}\right)
\end{aligned}
$$

REMARK 3.1. The treatment of error term $N$, above, we regard as the most delicate and novel aspect of our argument. Without complete cancellation of offdiagonal terms, we see no way that such a "Goodman-type" estimate can close, due to exponential growth in $C_{*}$ of $\sup |\alpha|$.

Kawashima-type estimate. Next, we perform a "Kawashima-type" estimate of the type formalized in [17]. Applying (2.3) to $W=U_{x}$ and $K=\tilde{K}_{\alpha}$,

$$
\frac{1}{2}\left\langle U_{x x}, \tilde{K}_{\alpha} U_{x}\right\rangle_{t}=\left\langle U_{x x}, \tilde{K}_{\alpha} U_{x t}\right\rangle+\frac{1}{2}\left\langle U_{x x},\left(\tilde{K}_{\alpha}\right)_{t} U_{x}\right\rangle+\frac{1}{2}\left\langle U_{x},\left(\tilde{K}_{\alpha}\right)_{x} U_{x t}\right\rangle
$$

Thanks to (3.7) and to Young's inequality, the second term is easily bounded by

$$
\frac{1}{2}\left\langle U_{x x},\left(\tilde{K}_{\alpha}\right)_{t} U_{x}\right\rangle \leq C \bar{\zeta}\left|U_{x x}\right|_{L^{2}}^{2}+C\left(\bar{\zeta}, C_{*}\right)\left|U_{x}\right|_{L^{2}}^{2}
$$

with $\bar{\zeta}$ as in previously chosen.

Differentiating with respect to $x$ equation (3.1), we obtain

$$
U_{x t}+\left(\tilde{A} U_{x}\right)_{x}-(\tilde{Q} U)_{x}=\left(M_{1}(U) \bar{U}_{x}\right)_{x}+\left(0, I_{r}\right)^{t} M_{2}(U)_{x}+\dot{\delta}(t)\left(\bar{U}_{x x}+U_{x x}\right),
$$


hence (twice) the last term in (3.26) can be rewritten as

$$
\begin{aligned}
&\left\langle U_{x},\left(\tilde{K}_{\alpha}\right)_{x} U_{x t}\right\rangle=-\left\langle U_{x},\left(\tilde{K}_{\alpha}\right)_{x} \frac{d \tilde{A}}{d \tilde{U}}\left(\bar{U}_{x}+U_{x}\right) U_{x}\right\rangle-\left\langle U_{x},\left(\tilde{K}_{\alpha}\right)_{x} \tilde{A} U_{x x}\right\rangle \\
&+\left\langle U_{x},\left(\tilde{K}_{\alpha}\right)_{x} \frac{d \tilde{Q}}{d \tilde{U}}\left(\bar{U}_{x}+U_{x}\right) U_{x}\right\rangle+\left\langle U_{x},\left(\tilde{K}_{\alpha}\right)_{x} \tilde{Q} U_{x}\right\rangle \\
&+\left\langle U_{x},\left(\tilde{K}_{\alpha}\right)_{x} \frac{d M_{1}}{d U} U_{x} \bar{U}_{x}\right\rangle+\left\langle U_{x},\left(\tilde{K}_{\alpha}\right)_{x} M_{1}(U) \bar{U}_{x x}\right\rangle \\
&+\left\langle U_{x},\left(\tilde{K}_{\alpha}\right)_{x}\left(0, I_{r}\right)^{t} \frac{d M_{2}}{d U} U_{x}\right\rangle+\dot{\delta}(t)\left\langle U_{x},\left(\tilde{K}_{\alpha}\right)_{x}\left(\bar{U}_{x x}+U_{x x}\right)\right\rangle
\end{aligned}
$$

All of the terms not containing $U_{x x}$ can be estimated by $C\left(C_{*}\right)\left(|U|_{H^{1}}^{2}+|\dot{\delta}(t)|^{2}\right)$ (using once more (3.7)). For the remaining terms,

$$
-\left\langle U_{x},\left(\tilde{K}_{\alpha}\right)_{x} \tilde{A} U_{x x}\right\rangle+\dot{\delta}(t)\left\langle U_{x},\left(\tilde{K}_{\alpha}\right)_{x} U_{x x}\right\rangle \leq C \bar{\zeta}\left|U_{x x}\right|_{L^{2}}^{2}+C\left(C_{*}, \bar{\zeta}\right)\left|U_{x}\right|_{L^{2}}^{2}
$$

Hence,

$$
\frac{1}{2}\left\langle U_{x},\left(\tilde{K}_{\alpha}\right)_{x} U_{x t}\right\rangle \leq C \bar{\zeta}\left|U_{x x}\right|_{L^{2}}^{2}+C\left(C_{*}, \bar{\zeta}\right)\left(|U|_{H^{1}}^{2}+|\dot{\delta}(t)|^{2}\right)
$$

Inserting (3.28), the first term on the righthand side of (3.26) becomes

$$
\begin{aligned}
&\left\langle U_{x x}, \tilde{K}_{\alpha} U_{x t}\right\rangle=-\left\langle U_{x x}, \tilde{K}_{\alpha} \frac{d \tilde{A}}{d \tilde{U}}\left(\bar{U}_{x}+U_{x}\right) U_{x}\right\rangle-\left\langle U_{x x}, \tilde{K}_{\alpha} \tilde{A} U_{x x}\right\rangle \\
&+\left\langle U_{x x}, \tilde{K}_{\alpha} \frac{d \tilde{Q}}{d \tilde{U}}\left(\bar{U}_{x}+U_{x}\right) U_{x}\right\rangle+\left\langle U_{x x}, \tilde{K}_{\alpha} \tilde{Q} U_{x}\right\rangle \\
&+\left\langle U_{x x}, \tilde{K}_{\alpha} \frac{d M_{1}}{d U} U_{x} \bar{U}_{x}\right\rangle+\left\langle U_{x x}, \tilde{K}_{\alpha} M_{1}(U) \bar{U}_{x x}\right\rangle \\
&+\left\langle U_{x x}, \tilde{K}_{\alpha}\left(0, I_{r}\right)^{t} \frac{d M_{2}}{d U} U_{x}\right\rangle+\dot{\delta}(t)\left\langle U_{x x},\left(\tilde{K}_{\alpha}\right)_{x}\left(\bar{U}_{x x}+U_{x x}\right)\right\rangle .
\end{aligned}
$$

The terms containing at least one of $U_{x}, U, \dot{\delta}$ can be estimated (applying Young's inequality) by $C(\bar{\zeta}+\zeta)\left|U_{x x}\right|_{L^{2}}^{2}+C\left(\bar{\zeta}, C_{*}\right)\left(|U|_{H^{1}}^{2}+|\dot{\delta}(t)|^{2}\right)$; hence,

$$
\begin{array}{r}
\left\langle U_{x x}, \tilde{K}_{\alpha} U_{x t}\right\rangle \leq-\left\langle U_{x x}, \tilde{K}_{\alpha} \tilde{A} U_{x x}\right\rangle+C(\bar{\zeta}+\zeta)\left|U_{x x}\right|_{L^{2}}^{2} \\
+C\left(\bar{\zeta}, C_{*}\right)\left(|U|_{H^{1}}^{2}+|\dot{\delta}(t)|^{2}\right) .
\end{array}
$$

Finally, (3.26), (3.27), (3.29) and (3.30) give

$$
\begin{array}{r}
\frac{1}{2}\left\langle U_{x x}, \tilde{K}_{\alpha} U_{x}\right\rangle_{t} \leq-\left\langle U_{x x}, \tilde{K}_{\alpha} \tilde{A} U_{x x}\right\rangle+C(\bar{\zeta}+\zeta)\left|U_{x x}\right|_{L^{2}}^{2} \\
+C\left(C_{*}, \bar{\zeta}\right)\left(|U|_{H^{1}}^{2}+|\dot{\delta}(t)|^{2}\right) .
\end{array}
$$


Adding (3.25) and (3.31), we obtain

$$
\begin{array}{r}
\frac{1}{2}\left(\left\langle\tilde{A}_{\alpha}^{0} U_{x x}, U_{x x}\right\rangle_{t}+\left\langle U_{x x}, \tilde{K}_{\alpha} U_{x}\right\rangle\right)_{t} \leq-\frac{1}{2}\left\langle U_{x x},\left(\tilde{A}^{0}\right)^{\frac{1}{2}} \tilde{O}\left(\alpha_{x} \tilde{D}\right) \tilde{O}^{t}\left(\tilde{A}^{0}\right)^{\frac{1}{2}} U_{x x}\right\rangle \\
+C\left\langle U_{x x},\left|\bar{U}_{x}\right| \tilde{A}_{\alpha}^{0} U_{x x}\right\rangle+\left\langle U_{x x},\left(-\tilde{K}_{\alpha} \tilde{A}+\tilde{A}_{\alpha}^{0} \tilde{Q}+\left(\tilde{A}^{0}\right)^{\frac{1}{2}} \tilde{O} N \tilde{O}^{t}\left(\tilde{A}^{0}\right)^{\frac{1}{2}}\right) U_{x x}\right\rangle \\
+C\left(C_{*}\right)(\zeta+\bar{\zeta})\left|U_{x x}\right|_{L^{2}}^{2}+C\left(C_{*}, \bar{\zeta}\right)\left(|U|_{H^{1}}^{2}+|\dot{\delta}(t)|^{2}\right) .
\end{array}
$$

Recalling (3.8) and (3.9), we obtain, finally,

$$
\begin{array}{r}
\left(\left\langle\tilde{A}^{0} U_{x x}, U_{x x}\right\rangle+\left\langle U_{x x}, \tilde{K}_{\alpha} U_{x}\right\rangle\right)_{t} \leq-\left(\theta C_{*}-C\right)\left\langle U_{x x},\left|\bar{U}_{x}\right| \tilde{A}_{\alpha}^{0} U_{x x}\right\rangle \\
+\left(-\theta\left(C_{*}\right)+C\left(C_{*}\right)(\zeta+\bar{\zeta})\right)\left|U_{x x}\right|_{L^{2}}^{2}+C\left(\bar{\zeta}, C_{*}\right)\left(\left.\left|U_{H^{1}}^{2}+\right| \dot{\delta}(t)\right|^{2}\right) \\
\leq-\frac{1}{2} \theta\left(C_{*}\right)\left|U_{x x}\right|_{L^{2}}^{2}+C\left(\bar{\zeta}, C_{*}\right)\left(|U|_{H^{1}}^{2}+|\dot{\delta}(t)|^{2}\right)
\end{array}
$$

provided that $C_{*}$ is taken sufficiently large and $\bar{\zeta}$, $\zeta$ sufficiently small that $C \leq \theta C_{*}$ and $-\theta\left(C_{*}\right)+C\left(C_{*}\right)(\zeta+\bar{\zeta}) \leq-\theta\left(C_{*}\right) / 2$.

Given $M>0$, let us set

$$
\mathcal{E}(U):=\left\langle\tilde{A}^{0} U_{x x}, U_{x x}\right\rangle+\left\langle U_{x x}, \tilde{K}_{\alpha} U_{x}\right\rangle+M|U|_{L^{2}}^{2} .
$$

Since, for $U \in H^{2},\left|U_{x}\right|_{L^{2}}$ can be bounded by $C\left(|U|_{L^{2}}+\left|U_{x x}\right|_{L^{2}}\right)$ for some $C>0$, then the functional defined in (3.34) is equivalent to $|U|_{H^{2}}^{2}$ if $M$ is large enough. Moreover, from (3.1) it follows that

$$
\frac{d|U|_{L^{2}}^{2}}{d t} \leq C\left(|U|_{H^{1}}^{2}+|\dot{\delta}(t)|^{2}\right)
$$

Therefore,

$$
\frac{d \mathcal{E}}{d t} \leq-\frac{1}{2} \theta\left(C_{*}\right)\left|U_{x x}\right|_{L^{2}}^{2}+C\left(|U|_{H^{1}}^{2}+|\dot{\delta}(t)|^{2}\right)
$$

for some $C>0$. Passing through Fourier transform, it is easy to see that, for $U \in H^{2}$ there holds

$$
\left|U_{x}\right|_{L^{2}}^{2} \leq C|U|_{L^{2}}^{2}+\frac{1}{C}\left|U_{x x}\right|_{L^{2}}^{2} \quad \forall C>0 .
$$

Hence, using $\mathcal{E} \geq C\left|U_{x x}\right|_{L^{2}}^{2}$ and choosing $C$ big enough in (3.35), we get

$$
\frac{d \mathcal{E}}{d t} \leq-\theta \mathcal{E}+C\left(|U|_{L^{2}}^{2}+|\dot{\delta}(t)|^{2}\right)
$$

for some $C, \theta>0$. Multiplying by $e^{\theta t}$ and integrating in time from 0 to $t$, we get (1.8) and the proof of Proposition 1.4 is complete.

REMARK 3.2. The energy estimate (1.9) can be deduced from (3.33) as follows. Integrating (3.33) from 0 to $t$ yields

$$
\begin{array}{r}
\left.\left(\left\langle\tilde{A}_{\alpha}^{0} U_{x x}, U_{x x}\right\rangle+\left\langle U_{x x}, \tilde{K}_{\alpha} U_{x}\right\rangle\right)\right|_{0} ^{t}+\theta\left(C_{*}\right) \int_{0}^{t}\left|U_{x x}\right|_{L^{2}}^{2}(s) d s \\
\leq C\left(C_{*}, \bar{\zeta}\right) \int_{0}^{t}\left(|U|_{H^{1}}^{2}+|\dot{\delta}|^{2}\right)(s) d s
\end{array}
$$


Rearranging, using positive definiteness of $\tilde{A}_{\alpha}^{0}$, using Young's inequality to bound

$$
\left\langle U_{x x}, K_{\alpha} U_{x}\right\rangle(t) \leq \bar{\zeta}\left|U_{x x}\right|_{L^{2}}^{2}(t)+C \bar{\zeta}^{-1}\left|U_{x}\right|_{L^{2}}^{2}(t) \leq \bar{\zeta}\left|U_{x x}\right|_{L^{2}}^{2}(t)+C \bar{\zeta}^{-1} \zeta^{2}
$$

and recalling, by assumption, that $\left(\left\langle\tilde{A}_{\alpha}^{0} U_{x}, U_{x}\right\rangle+\left\langle U_{x}, \tilde{K}_{\alpha} U\right\rangle\right)(0) \leq C \zeta^{2}$, we obtain (1.9) as claimed.

4. Nonlinear stability. We now establish Theorem 1.2 on nonlinear stability.

Linearized estimates. Linearizing (1.2) about the stationary solution $(\bar{u}, \bar{v})$, we obtain the linearized equations

$$
U_{t}=\mathcal{L} U:=-(A U)_{x}+Q U
$$

where

$$
A:=\left(\begin{array}{c}
d f \\
d g
\end{array}\right)(\bar{u}, \bar{v}), \quad Q:=\left(\begin{array}{c}
0 \\
d q(\bar{u}, \bar{v})
\end{array}\right) \quad \text { and } \quad U:=\left(\begin{array}{c}
u \\
v
\end{array}\right) \quad\left(u \in \mathbb{R}^{n}, v \in \mathbb{R}^{r}\right) .
$$

Define the associated Green distribution $G(x, t ; y)$ by

$$
\left(\partial_{t}-\mathcal{L}\right) G(x, t ; y)=\delta_{(y, 0)}(x, t) .
$$

We have the following bounds established in Proposition 1.11 and Lemmas 7.1-7.5 of [24]. (See also "notes" below (7.22) of [24], which is used in the short-time estimate for $\left.\left|e_{y}(\cdot, t)\right|_{L^{p}} \cdot\right)$

Proposition 4.1 ([24]). Assuming (A1)-(A2) and (HO)-(H5), the Green distribution $G$ may be decomposed as

$$
G=E+\tilde{G}+H
$$

where $E(x, t ; y)=e(y, t) \bar{U}_{x}(x)$, with

$$
\begin{gathered}
\left|e_{y}(\cdot, t)\right|_{L^{p}} \leq C t^{\frac{1}{2}}(1+t)^{-\frac{1}{2}\left(1-\frac{1}{p}\right)-\frac{1}{2}}, \quad\left|e_{t}(\cdot, t)\right|_{L^{p}} \leq C t^{-\frac{1}{2}\left(1-\frac{1}{p}\right)} \\
\left|e_{t y}(\cdot, t)\right|_{L^{p}} \leq C t^{-\frac{1}{2}\left(1-\frac{1}{p}\right)-\frac{1}{2}}, \\
\left|\int_{\mathbb{R}} \tilde{G}(\cdot, t ; y) f(y) d y\right|_{L^{p}} \leq C(1+t)^{-\frac{1}{2}\left(1-\frac{1}{r}\right)}|f|_{L^{q}} \\
\left|\int_{\mathbb{R}} \tilde{G}(\cdot, t ; y)\left(0, I_{r}\right)^{t} f(y) d y\right|_{L^{p}} \leq C(1+t)^{-\frac{1}{2}\left(1-\frac{1}{r}\right)-\frac{1}{2}|f|_{L^{q}}} \\
\left|\int_{\mathbb{R}} \tilde{G}_{y}(\cdot, t ; y) f(y) d y\right|_{L^{p}} \leq C(1+t)^{-\frac{1}{2}\left(1-\frac{1}{r}\right)-\frac{1}{2}|f|_{L^{q}}+C e^{-\eta t}|f|_{L^{p}}}
\end{gathered}
$$

and

$$
\left|\int_{\mathbb{R}} H(\cdot, t ; y) f(y) d y\right|_{L^{p}} \leq C e^{-\eta t}|f|_{L^{p}}
$$


for all $t \geq 0$, some $C, \eta>0$, for any $1 \leq r \leq p$ and $f \in L^{q}$ (resp. $L^{p}$ ), where $1 / r+1 / q=1+1 / p$.

Here, the "excited" component $E$ accounts for contributions in the direction of the translational zero eigenfunction $\bar{U}_{x}$, while the "hyperbolic" component $H$ accounts for propagation of signals along along hyperbolic characteristics, its time-exponential damping a consequence of the genuine coupling condition (1.3). The reduced Green distribution $\tilde{G}$, accounting for long-time behavior in the far fields, is approximately a sum of Gaussian signals scattered by the shock layer. For further discussion, see [24].

Proof of Theorem 1.2. We first treat the case $p \geq 2$, afterward extending to $p \leq 2$ bya bootstrap argument.

$L^{p}$ stability, $2 \leq p \leq \infty$. Define the nonlinear perturbation

$$
U(x, t):=\left(\begin{array}{l}
u \\
v
\end{array}\right)(x+\delta(t), t)-\left(\begin{array}{l}
\bar{u} \\
\bar{v}
\end{array}\right)(x)=\tilde{U}(x+\delta(t), t)-\bar{U}(x),
$$

where $\delta(t)$ (estimating shock location) is to be determined later; for definiteness, fix $\delta(0)=0$. Then,

$$
U_{t}-\mathcal{L} U=N_{1}(U)_{x}+\left(0, I_{r}\right)^{t} N_{2}(U)+\dot{\delta}(t)\left(\bar{U}_{x}+U_{x}\right),
$$

where

$$
N_{j}(U, U)=\mathcal{O}\left(|U|^{2}\right) \quad \text { and } \quad N_{j}(U, U)_{x}=\mathcal{O}\left(|U|\left|U_{x}\right|\right)
$$

so long as $|U|$ remains bounded. By Duhamel's principle, and the fact that

$$
\int_{\mathbb{R}} G(x, t ; y) \bar{U}_{x}(y) d y=e^{\mathcal{L} t} \bar{U}_{x}(x)=\bar{U}_{x}(x),
$$

we have

$$
\begin{array}{r}
U(x, t)=\int_{\mathbb{R}} G(x, t ; y) U_{0}(y) d y-\int_{0}^{t} \int_{\mathbb{R}} G_{y}(x, t-s ; y)\left(N_{1}(U)+\dot{\delta} U\right)(y, s) d y d s \\
+\int_{0}^{t} \int_{\mathbb{R}} G(x, t-s ; y)\left(0, I_{r}\right)^{t} N_{2}(U)(y, s) d y d s+\delta(t) \bar{U}_{x} .
\end{array}
$$

Defining the instantaneous shock location

$$
\delta(t)=-\int_{\mathbb{R}} e(y, t) U_{0}(y) d y+\int_{0}^{t} \int_{\mathbb{R}} e_{y}(y, t-s)\left(N_{1}(U)+\dot{\delta} U\right)(y, s) d y d s,
$$

where $E, e$ are defined as in Proposition 4.1 and recalling decomposition (4.2), we thus obtain the reduced equations:

$$
\begin{aligned}
U(x, t) & =\int_{\mathbb{R}}(H+\tilde{G})(x, t ; y) U_{0}(y) d y \\
& +\int_{0}^{t} \int_{\mathbb{R}} H(x, t-s ; y)\left(N_{1}(U)_{x}+\left(0, I_{r}\right)^{t} N_{2}(U)+\dot{\delta} U_{x}\right)(y, s) d y d s \\
& -\int_{0}^{t} \int_{\mathbb{R}} \tilde{G}_{y}(x, t-s ; y)\left(N_{1}(U)+\dot{\delta} U\right)(y, s) d y d s \\
& +\int_{0}^{t} \int_{\mathbb{R}} \tilde{G}(x, t-s ; y)\left(0, I_{r}\right)^{t} N_{2}(U)(y, s) d y d s,
\end{aligned}
$$


and, differentiating (4.3) with respect to $t$, and using $\left|e_{y}(\cdot, s)\right|_{L^{1}} \rightarrow 0$ as $t \rightarrow 0$,

$$
\dot{\delta}(t)=-\int_{\mathbb{R}} e_{t}(y, t) U_{0}(y) d y+\int_{0}^{t} \int_{\mathbb{R}} e_{y t}(y, t-s)\left(N_{1}(U)+\dot{\delta} U\right)(y, s) d y d s .
$$

Define

$$
\zeta(t):=\sup _{0 \leq s \leq t, 2 \leq p \leq \infty}\left[|U(\cdot, s)|_{L^{p}}(1+s)^{\frac{1}{2}\left(1-\frac{1}{p}\right)}+|\dot{\delta}(s)|(1+s)^{\frac{1}{2}}+|\delta(s)|\right] .
$$

We shall establish:

ClaIM. For all $t \geq 0$ for which a solution exists with $\zeta$ uniformly bounded by some fixed, sufficiently small constant, there holds

$$
\zeta(t) \leq C_{2}\left(\left|U_{0}\right|_{L^{1} \cap H^{2}}+\zeta(t)^{2}\right)
$$

From this result, it follows by continuous induction that, provided $\left|U_{0}\right|_{L^{1} \cap H^{2}}<1 / 4 C_{2}^{2}$, there holds

$$
\zeta(t) \leq 2 C_{2}\left|U_{0}\right|_{L^{1} \cap H^{2}}
$$

for all $t \geq 0$ such that $\zeta$ remains small. For, by standard short-time theory/local wellposedness in $H^{2}$, and the standard principle of continuation, there exists a solution $U(\cdot, t) \in H^{2}$ on the open time-interval for which $|U|_{H^{2}}$ remains bounded, and on this interval $\zeta$ is well-defined and continuous. Now, let $[0, T)$ be the maximal interval on which $|U|_{H^{2}}$ remains strictly bounded by some fixed, sufficiently small constant $\delta>0$. By Proposition 1.4, and the one-dimensional Sobolev bound $|U|_{W^{1, \infty}} \leq C|U|_{H^{2}}$, we have

$$
\begin{aligned}
|U(t)|_{H^{2}}^{2} & \leq C|U(0)|_{H^{2}}^{2} e^{-\theta t}+C \int_{0}^{t} e^{-\theta_{2}(t-\tau)}\left(|U|_{L^{2}}^{2}+|\dot{\delta}|^{2}\right)(\tau) d \tau \\
& \leq C_{2}\left(|U(0)|_{H^{2}}^{2}+\zeta(t)^{2}\right)(1+t)^{-\frac{1}{2}}
\end{aligned}
$$

and so the solution continues so long as $\zeta$ remains small, with bound (4.7), at once yielding existence and the claimed sharp $L^{p} \cap H^{2}$ bounds, $2 \leq p \leq \infty$.

Thus, it remains only to establish the claim above.

Proof of Claim. We must show that each of the quantities $|U|_{L^{p}}(1+s)^{\frac{1}{2}\left(1-\frac{1}{p}\right)}$, $|\dot{\delta}|(1+s)^{\frac{1}{2}}$, and $|\delta|$ is separately bounded by

$$
C\left(\left|U_{0}\right|_{L^{1} \cap H^{2}}+\zeta(t)^{2}\right)
$$

for some $C>0$, all $0 \leq s \leq t$, so long as $\zeta$ remains sufficiently small. By (4.4)-(4.5), 
we have

$$
\begin{aligned}
|U|_{L^{p}}(t) \leq & \left|\int_{\mathbb{R}}(H+\tilde{G})(x, t ; y) U_{0}(y) d y\right|_{L^{p}} \\
& +\left|\int_{0}^{t} \int_{\mathbb{R}} H(x, t-s ; y) N_{1}(U)_{y}(y, s) d y d s\right|_{L^{p}} \\
& +\left|\int_{0}^{t} \int_{\mathbb{R}} H(x, t-s ; y)\left(0, I_{r}\right)^{t} N_{2}(U)(y, s) d y d s\right|_{L^{p}} \\
& +\left|\int_{0}^{t} \int_{\mathbb{R}} H(x, t-s ; y) \dot{\delta} U_{x}(y, s) d y d s\right|_{L^{p}} \\
& +\left|\int_{0}^{t} \int_{\mathbb{R}} \tilde{G}_{y}(x, t-s ; y) N_{1}(U)(y, s) d y d s\right|_{L^{p}} \\
& +\left|\int_{0}^{t} \int_{\mathbb{R}} \tilde{G}(x, t-s ; y)\left(0, I_{r}\right)^{t} N_{2}(U)(y, s) d y d s\right|_{L^{p}} \\
& +\left|\int_{0}^{t} \int_{\mathbb{R}} \tilde{G}_{y}(x, t-s ; y) \dot{\delta} U(y, s) d y d s\right|_{L^{p}} \\
=: & I_{a}+I_{b}+I_{c}+I_{d}+I_{e}+I_{f}+I_{g},
\end{aligned}
$$

$$
|\dot{\delta}|(t) \leq\left|\int_{\mathbb{R}} e_{t}(y, t) U_{0}(y) d y\right|+\left|\int_{0}^{t} \int_{\mathbb{R}} e_{y t}(y, t-s) \dot{\delta} U(y, s) d y d s\right|=: I I_{a}+I I_{b}
$$

and

$$
|\delta|(t) \leq\left|\int_{\mathbb{R}} e(y, t) U_{0}(y) d y\right|+\left|\int_{0}^{t} \int_{\mathbb{R}} e_{y}(y, t-s) \dot{\delta} U(y, s) d y d s\right|=: I I I_{a}+I I I_{b}
$$

We estimate each term in turn, following the approach of [37, 24, 25, 27]. Applying the bounds of Proposition 4.1, we find that the linear term $I_{a}$ satisfies

$$
\begin{aligned}
I_{a} & \leq|U|_{L^{p}}(t)\left|\int_{\mathbb{R}^{R}} H U_{0} d y\right|_{L^{p}}+\left|\int_{\mathbb{R}} \tilde{G} U_{0} d y\right|_{L^{p}} \\
& \leq C e^{-\theta t}\left|U_{0}\right|_{L^{p}}+C(1+t)^{-\frac{1}{4}}\left|U_{0}\right|_{L^{1}} \leq C\left|U_{0}\right|_{L^{1} \cap H^{2}}(1+t)^{-\frac{1}{4}}
\end{aligned}
$$

Likewise, applying the bounds of Proposition 4.1 together with definition (4.6) and energy estimate (4.8), we have:

$$
\begin{aligned}
& I_{b}=\left|\int_{0}^{t} \int_{\mathbb{R}} H N_{1}(U)_{y} d y d s\right|_{L^{p}} \leq C \int_{0}^{t} e^{-\eta(t-s)}|U|_{L^{\infty}}\left|U_{x}\right|_{L^{p}}(s) d s \\
& \leq C \int_{0}^{t} e^{-\eta(t-s)}|U|_{L^{\infty}}|U|_{H^{2}}(s) d s \leq C \zeta(t)^{2} \int_{0}^{t} e^{-\eta(t-s)}(1+s)^{-\frac{3}{4}} d s \\
& \leq C \zeta(t)^{2}(1+t)^{-\frac{3}{4}}, \\
& I_{c}=\left|\int_{0}^{t} \int_{\mathbb{R}} H\left(0, I_{r}\right)^{t} N_{2}(U) d y d s\right|_{L^{p}} \leq C \int_{0}^{t} e^{-\eta(t-s)}|U|_{L^{\infty}}|U|_{L^{p}}(s) d s \\
& \leq C \zeta(t)^{2} \int_{0}^{t} e^{-\eta(t-s)}(1+s)^{-\frac{3}{4}} d s \leq C \zeta(t)^{2}(1+t)^{-\frac{3}{4}}
\end{aligned}
$$




$$
\begin{aligned}
I_{d}=\left|\int_{0}^{t} \int_{\mathbb{R}} H \dot{\delta} U_{x} d y d s\right|_{L^{p}} \leq C \int_{0}^{t} e^{-\eta(t-s)}|\dot{\delta}|\left|U_{x}\right|_{L^{p}}(s) d s \\
\quad \leq C \zeta(t)^{2} \int_{0}^{t} e^{-\eta(t-s)}(1+s)^{-\frac{3}{4}} d s \leq C \zeta(t)^{2}(1+t)^{-\frac{3}{4}}
\end{aligned}
$$

(Proposition 4.1, $2 \leq p=q \leq \infty, r=1$ ) and

$$
\begin{aligned}
I_{e} & =\left|\int_{0}^{t} \int_{\mathbb{R}} \tilde{G}_{y} N_{1}(U) d y d s\right|_{L^{p}} \leq C \int_{0}^{t}(1+(t-s))^{-\frac{1}{2}\left(1-\frac{1}{p}\right)-\frac{1}{4}}|U|_{L^{\infty}}|U|_{L^{2}}(s) d s \\
& \leq C \zeta(t)^{2} \int_{0}^{t}(1+(t-s))^{-\frac{1}{2}\left(1-\frac{1}{p}\right)-\frac{1}{4}}(1+s)^{-\frac{3}{4}} d s \leq C \zeta(t)^{2}(1+t)^{-\frac{1}{2}\left(1-\frac{1}{p}\right)}, \\
I_{f}= & \left|\int_{0}^{t} \int_{\mathbb{R}} \tilde{G}\left(0, I_{r}\right)^{t} N_{2}(U) d y d s\right|_{L^{p}} \leq C \int_{0}^{t}(1+(t-s))^{-\frac{1}{2}\left(1-\frac{1}{p}\right)-\frac{1}{4}}|U|_{L^{\infty}}|U|_{L^{2}}(s) d s \\
\leq & C \zeta(t)^{2} \int_{0}^{t}(1+(t-s))^{-\frac{1}{2}\left(1-\frac{1}{p}\right)-\frac{1}{4}}(1+s)^{-\frac{3}{4}} d s \leq C \zeta(t)^{2}(1+t)^{-\frac{1}{2}\left(1-\frac{1}{p}\right)}, \\
I_{g}= & \left|\int_{0}^{t} \int_{\mathbb{R}} \tilde{G}_{y} \dot{\delta} U d y d s\right|_{L^{p}} \leq C \int_{0}^{t}(1+(t-s))^{-\frac{1}{2}\left(1-\frac{1}{p}\right)-\frac{1}{4}}|\dot{\delta}||U|_{L^{2}}(s) d s \\
\leq & C \zeta(t)^{2} \int_{0}^{t}(1+(t-s))^{-\frac{1}{2}\left(1-\frac{1}{p}\right)-\frac{1}{4}}(1+s)^{-\frac{3}{4}} d s \leq C \zeta(t)^{2}(1+t)^{-\frac{1}{2}\left(1-\frac{1}{p}\right)},
\end{aligned}
$$

(Proposition 4.1, $2 \leq p \leq \infty, q=2$ ). Summing bounds (4.12)-(4.18), we obtain the desired bound on $|U|_{L^{p}}$.

Similarly, applying the bounds of Proposition 4.1 together with definition (4.6), we find that

$$
I I_{a}=\left|\int_{\mathbb{R}} e_{t} U_{0} d y\right| \leq\left|e_{t}(y, t)\right|_{L^{\infty}}(t)\left|U_{0}\right|_{L^{1}} \leq C\left|U_{0}\right|_{L^{1}}(1+t)^{-\frac{1}{2}}
$$

and

$$
\begin{aligned}
& I I_{b}=\left|\int_{0}^{t} \int_{\mathbb{R}} e_{y t} \dot{\delta} U d y d s\right| \leq \int_{0}^{t}\left|e_{y t}\right|_{L^{2}}(t-s)|\dot{\delta}||U|_{L^{2}}(s) d s \\
& \leq C \zeta(t)^{2} \int_{0}^{t}(t-s)^{-3 / 4}(1+s)^{-\frac{3}{4}} d s \leq C \zeta(t)^{2}(1+t)^{-\frac{1}{2}}
\end{aligned}
$$

while

$$
I I I_{a}=\left|\int_{\mathbb{R}} e U_{0} d y\right| \leq|e(y, t)|_{L^{\infty}}(t)\left|U_{0}\right|_{L^{1}} \leq C\left|U_{0}\right|_{L^{1}}
$$

and

$$
\begin{aligned}
I I I_{b}=\left|\int_{0}^{t} \int_{\mathbb{R}} e_{y} \dot{\delta} U d y d s\right| \leq & \int_{0}^{t}\left|e_{y}\right|_{L^{2}}(t-s)|\dot{\delta}||U|_{L^{2}}(s) d s \\
& \leq C \zeta(t)^{2} \int_{0}^{t}(t-s)^{-\frac{1}{4}}(1+s)^{-\frac{3}{4}} d s \leq C \zeta(t)^{2} .
\end{aligned}
$$


Summing (4.19)-(4.20) and (4.21)-(4.22), we obtain the desired bounds on $\dot{\delta}$ and $\delta$.

This completes the proof of the claim, giving the result for $2 \leq p \leq \infty$.

$L^{p}$ stability, $1 \leq p \leq 2$. The source term $\dot{\delta} U_{x}$ appearing in the reduced equations is convenient for high norm estimates $L^{p}, p \geq 2$, but (since it would lead to a source term involving higher derivative factor $\left|U_{x}\right|_{L^{p}}$ not controlled by energy estimates) not for low norm estimates $L^{p}, 1 \leq p<2$. To treat low norms, we redefine

$$
U:=\tilde{U}(x, t)-\bar{U}(x-\delta(t))
$$

following [40], which has the effect of replacing $\dot{\delta} U_{x}$ in the reduced equations with "centering errors"

$$
\begin{aligned}
S_{1}(\delta, \dot{\delta}, U)_{x}+\left(\begin{array}{c}
0 \\
I_{r}
\end{array}\right) S_{2}(\delta):= & -\left(\left(A \left(\bar{U}(x-\delta)-A(\bar{U}(x)) U+\dot{\delta}(\bar{U}(x-\delta)-\bar{U}(x))_{x}\right.\right.\right. \\
& +\delta(Q(\bar{U}(x-\delta)-Q(\bar{U}(x)) U
\end{aligned}
$$

satisfying

$$
\begin{gathered}
\left|S_{1}(\delta, \dot{\delta}, U)(y, s)\right| \leq|\delta|(|U|+|\dot{\delta}|) e^{-\theta|y|} \\
\left|S_{1}(\delta, \dot{\delta}, U)_{x}(y, s)\right| \leq|\delta|\left(|U|+|\dot{\delta}|+\left|U_{x}\right|\right) e^{-\theta|y|} \\
\left|S_{2}(\delta)(y, s)\right| \leq|\delta||U| e^{-\theta|y|}
\end{gathered}
$$

and therefore

$$
\begin{aligned}
\left|S_{1}\right|_{L^{1}} & \leq\left(|U|_{L^{\infty}}+|\dot{\delta}|\right) \leq C(1+t)^{-\frac{1}{2}}\left|U_{0}\right|_{L^{1} \cap H^{2}} \\
\left|\left(S_{1}\right)_{x}\right|_{L^{1}} & \leq\left(|U|_{L^{\infty}}+|U|_{H^{1}}+|\dot{\delta}|\right) \leq C(1+t)^{-\frac{1}{4}\left|U_{0}\right|_{L^{1} \cap H^{2}}}
\end{aligned}
$$

and

$$
\left|S_{2}\right|_{L^{1}} \leq|U|_{L^{\infty}}|\delta| \leq C(1+t)^{-\frac{1}{2}}\left|U_{0}\right|_{L^{1} \cap H^{2}}
$$

by the previously-obtained $L^{\infty}$ and $H^{1}$ estimates, which are unaffected by a spatial shift. Likewise,

$$
\left|N_{1}(U)\right|_{W^{1,1}},\left|N_{2}(U)\right|_{L^{1}} \leq|U|_{H^{1}}^{2} \leq C(1+t)^{-\frac{1}{2}}\left|U_{0}\right|_{L^{1} \cap H^{2}} .
$$

Thus, expressing $U$ by Duhamel's formula similarly as in (4.9)-(4.11) and estimating nonlinear terms using the bounds of Proposition 4.1 with $p=1, q=1$, we readily obtain the sharp $L^{1}$ decay estimate

$$
|U|_{L^{1}} \leq C\left|U_{0}\right|_{L^{1} \cap H^{2}}
$$

and, by interpolation with the previously obtained $L^{2}$ bound, the sharp $L^{p}$ estimate, $1 \leq p \leq 2$ of $|U|_{L^{p}} \leq C(1+t)^{\frac{1}{2}\left(1-\frac{1}{p}\right)}\left|U_{0}\right|_{L^{1} \cap H^{2}}$ as claimed. We omit the details, which are entirely similar to those already carried out. 
5. The characteristic case. Finally, we briefly discuss the uniformly characteristic case in which (H1)(i) is violated for a shock profile of a discrete kinetic model. This cannot occur for the simplest examples of the Broadwell or Jin-Xin models, for the reason that it would violate the subcharacteristic condition

$$
a_{j}<a_{j}^{* \pm}<a_{j+r},
$$

which is in turn necessary for strict dissipativity, (A2); see, e.g., [33, 24, 26]. Indeed, this holds in general for models with the property that $r$ consecutive characteristics take on only two values $(r=2$ for Broadwell; $r=n / 2$ for Jin-Xin, but the total number of characteristic values is 2); for, the subcharacteristic condition (5.1) then implies that $a_{p}^{* \pm}$ lie between the neighboring characteristic values $a_{p}$ and $a_{p+r}$, whence, by the Lax condition $a_{p}^{*-}>s>a_{p}^{*+}$, the speed $s$ does as well.

This is clearly an accident of low dimension, however, and for general models there is no physical reason that (H1)(i) should be satisfied. Indeed, though it is evidently satisfied generically, there is ample reason to discard this hypothesis. For, discretizing the Boltzmann equations

$$
f_{t}+\xi f_{x}=Q(\xi, f), \quad \xi \in \mathbb{R}^{1},
$$

by velocity $\xi$, where $\xi$ denotes velocity, $f(\xi, x)$ the probability distribution of speeds at spatial location $x$, and $Q(\xi, f)$ a collision term (local in $x$ but nonlocal in $\xi$ ), we find as the velocity mesh goes to zero that (H1)(i) is more and more poorly satisfied, so that uniformity of our estimates (or even the ball for which small-amplitude profiles are guaranteed to exist) is lost.

This is hardly the main difficulty in proceeding to the Boltzmann limit, which is rather the reverse problem of unboundedness of the multiplication operator $f \rightarrow \xi f$ (in our notation, blowup of the spectrum of $A$ ), and the associated lack of spectral gap between zero and the essential spectrum of the operator $A^{-1} Q$ appearing in the travelling-wave and eigenvalue ODE; see, e.g., [6]. Nonetheless, it is an issue that arises and should be addressed.

Fortunately, there is a simple fix, at least for discrete kinetic models. Namely, in case characteristics $a_{j}, \ldots, a_{k}$ coincide with shock speed $s$, we may substitute for (H1)(i) the more general hypotheses

$$
\operatorname{Re}\left(\begin{array}{lll}
L_{j} & \cdots & L_{k}
\end{array}\right) d Q\left(\begin{array}{c}
R_{j} \\
\vdots \\
R_{k}
\end{array}\right) \leq-\theta<0
$$

for some fixed left and right zero eigenbases $L_{i}$ and $R_{i}$, and

$$
\operatorname{ker} \tilde{A} \cap \operatorname{ker} d q=\emptyset,
$$

where without loss of generality $A$ is taken to be diagonal, $\tilde{A}:=A-s=d F-s$. This holds necessarily at $x= \pm \infty$ for some choice of diagonalizing transformation, by strict dissipativity, (A2), (recall that $\operatorname{Re} \sigma$ (block-diag $\left.\tilde{L} Q \tilde{R}_{ \pm}\right)<0$ follows from (A2) by Taylor expansion at infinity of the spectrum of the symbol $i \xi A_{ \pm}+Q_{ \pm}$; likewise, (A2) implies genuine coupling, (1.3), of which (5.3) is a weakened form), hence is always satisfied in the small-amplitude case. Whether or not it holds globally for physically interesting examples we do not know.

Review of the argument of Section 3 shows that auxiliary energy estimate (1.9) goes through under this hypothesis with constant weights $\alpha_{j}=\alpha_{j+1}=\cdots=\alpha_{k} \equiv 1$ 
in the zero-speed modes, since there are no error terms in these modes to be overcome and there is a uniformly good contribution by (5.2). Likewise, review of the arguments of [24] shows that the results obtained there carry though as well, with appropriate modification of the proofs. Namely, (5.2) and (5.3) together imply that

$$
\left(\begin{array}{c}
d f \\
d Q_{j} \\
\vdots \\
d Q_{k} \\
d g_{2}
\end{array}\right), \quad g=:\left(\begin{array}{c}
g_{1} \\
g_{2}
\end{array}\right), \quad g_{1} \in \mathbb{R}^{k-j+1}
$$

is full rank under some choice of coordinate system, whence we can again rewrite both traveling-wave and eigenvalue/resolvent equations as nondegenerate first-order systems and proceed as before. We omit the details as aside from our main purpose.

Note, in particular, that we obtain small-amplitude existence for fixed speed $s$ by a minor adjustment of the argument of [24] (namely, fixing the speed and letting endstates vary), without any assumption on the base state other than simplicity of the principal eigenvalue and strong dissipativity; this generalizes earlier results of $[34,24]$.

For $a_{j}, \ldots, a_{k}$ close to $s$, we may recover our previous results with uniform estimates by a singular perturbation version of the same argument. It would be interesting to extend this approach to more general relaxation models for which the speeds $a_{i}$ are not constant, in particular the case for which they are sometimes but not always characteristic.

\section{REFERENCES}

[1] G.I. Barenblatt, J. Garcia-Azorero, A. De Pablo, and J.L. Vazquez, Mathematical model of the non-equilibrium water-oil displacement in porous strata, Appl. Anal. 65 (1997), no.1-2, $19-45$.

[2] G.I. Barenblatt and A.P.Vinnichenko, Nonequilibrium filtration of nonmixing fluids, (russian) Adv. in Mech. 3 (1980), no.3, 35-50.

[3] A.A. Barmin and S.A. Egorushkin, Stability of shock waves, Adv. Mech. 15 (1992) No. 1-2, $3-37$.

[4] S. Benzoni-Gavage, D. Serre, and K. Zumbrun, Alternate Evans functions and viscous shock waves, SIAM J. Math. Anal. 32 (2001), no.5, 929-962.

[5] S. Bianchini, B.Hanouzet and R. Natalini, Asymptotic behavior of smooth solutions for weakly dissipative hyperbolic systems with a convex entropy in several space dimensions, in preparation.

[6] R. Caflisch and B. Nickolaenko, Shock profile solutions of the Boltzmann equation, Comm. Math. Phys. 86 (1982), no.2, 161-194.

[7] H. Freistühler and K. Zumbrun, Examples of unstable viscous shock waves, unpublished note, Institut für Mathematik, RWTH Aachen, February 1998.

[8] K.O. Friedrichs, Symmetric hyperbolic linear differential equations, Comm. Pure and Appl. Math. 7 (1954) 345-392.

[9] K.O. Friedrichs, On the laws of relativistic electro-magneto-fluid dynamics, Comm. Pure and Appl. Math. 27 (1974) 749-808.

[10] R. Gardner and K. Zumbrun, The Gap Lemma and geometric criteria for instability of viscous shock profiles, Comm. Pure Appl. Math. 51 (1998), no. 7, 797-855.

[11] P. Godillon, Linear stability of shock profiles for systems of conservation laws with semi-linear relaxation, Phys. D 148 (2001), no. 3-4, 289-316.

[12] J. Goodman, Remarks on the stability of viscous shock waves, in: Viscous profiles and numerical methods for shock waves (Raleigh, NC, 1990), 66-72, SIAM, Philadelphia, PA, (1991).

[13] P. Howard and K. Zumbrun, Pointwise estimates for dispersive-diffusive shock waves, Arch. Ration. Mech. Anal. 155 (2000), no.2, 85-169.

[14] P. Howard and K. Zumbrun, Stability of undercompressive shock profiles, preprint (2004). 
[15] J. Humpherys, Viscous-dispersive traveling waves I: admissibility and existence, preprint (2003).

[16] S. Jin and Z. Xin, The relaxation schemes for systems of conservation laws in arbitrary space dimensions, Comm. Pure Appl. Math. 48 (1995), no. 3, 235-276.

[17] S. Kawashima, Systems of a hyperbolic-parabolic composite type, with applications to the equations of magnetohydrodynamics, thesis, Kyoto University (1983).

[18] T. Kato, Perturbation theory for linear operators, Springer-Verlag, Berlin Heidelberg (1985).

[19] P.D. Lax, Hyperbolic systems of conservation laws and the mathematical theory of shock waves, Conference Board of the Mathematical Sciences Regional Conference Series in Applied Mathematics, No. 11. Society for Industrial and Applied Mathematics, Philadelphia, Pa., 1973. v+48 pp.

[20] T.-P. Liu, Nonlinear stability of shock waves for viscous conservation laws, Mem. Amer. Math. Soc. 56 (1985), no. 328, v+108 pp.

[21] A. Majda, The stability of multi-dimensional shock fronts - a new problem for linear hyperbolic equations, Mem. Amer. Math. Soc. 275 (1983).

[22] A. Majda, The existence of multi-dimensional shock fronts, Mem. Amer. Math. Soc. 281 (1983).

[23] A. Majda, Compressible fluid flow and systems of conservation laws in several space variables, Springer-Verlag, New York (1984), viii+ 159 pp.

[24] C. Mascia and K. Zumbrun, Pointwise Green's function bounds and stability of relaxation shocks, Indiana Univ. Math. J. 51 (2002), no.4, 773-904.

[25] C. Mascia and K. Zumbrun, Stability of small-amplitude shock profiles of symmetric hyperbolicparabolic systems, Comm. Pure Appl. Math. 57 (2004), no.7, 841-876.

[26] C. Mascia and K. Zumbrun, Pointwise Green function bounds for shock profiles of systems with real viscosity, Arch. Rational Mech. Anal. 169 (2003), no.3, 177-263.

[27] C. Mascia and K. Zumbrun, Stability of large-amplitude viscous shock profiles of hyperbolicparabolic systems, Arch. Ration. Mech. Anal. 172 (2004), no.1, 93-131.

[28] R. Plaza and K. Zumbrun, An Evans function approach to spectral stability of small-amplitude viscous shock profiles, preprint (2002).

[29] M-R. Raoofi, $L^{1}$ asymptotic behavior of viscous shock profiles of systems with real viscosity, preprint (2004)

[30] Y. Shizuta and S. Kawashima, Systems of equations of hyperbolic-parabolic type with applications to the discrete Boltzmann equation, Hokkaido Math. J. 14 (1985), no.2, 249-275.

[31] J.C. Strikwerda, Finite difference schemes and partial differential equations, (Chapman and Hall, New York (1989) xii+ 386 pp.

[32] G.B. Whitham, "Linear and nonlinear waves", Pure and Applied Mathematics, WileyInterscience [John Wiley \& Sons], New York-London-Sidney, 1974. xvi+636pp.

[33] W.-A. Yong, Basic properties of hyperbolic relaxation systems, Birkhauser's Series: Progress in Nonlinear Differential Equations and their Applications (2001), 207 pp.

[34] W.-A. Yong and K. Zumbrun, Existence of relaxation shock profiles for hyperbolic conservation laws, SIAM J. Appl. Math. 60 (2000), no. 5, 1565-1575.

[35] Y. Zeng, Gas dynamics in thermal nonequilibrium and general hyperbolic systems with relaxation, Arch. Ration. Mech. Anal. 150 (1999), no. 3, 225-279.

[36] K. Zumbrun, Stability of viscous shock waves, Lecture Notes, Indiana University (1998).

[37] K. Zumbrun, Refined wave-tracking and nonlinear stability of viscous Lax shocks, Methods. Appl. Anal. 7 (2000), no.4, 747-768.

[38] K. Zumbrun, Multidimensional stability of planar viscous shock waves, "Advances in the theory of shock waves", 307-516, Progr. Nonlinear Differential Equations Appl., 47, Birkhäuser Boston, Boston, MA, 2001.

[39] K. Zumbrun, Planar stability conditions for large-amplitude viscous shock profiles, lecture notes, CIME summer school: Cetraro, Italy, July 2003.

[40] K. Zumbrun, Stability of large-amplitude viscous shock profiles of the equations of fluid dynamics, to appear, Handbook of Fluids, Elsevier (2004).

[41] K.Zumbrun and P.Howard, Pointwise semigroup methods and stability of viscous shock waves, Indiana Univ. Math. J. 47 (1998), no.3, 741-871.

[42] K.Zumbrun and D.Serre, Viscous and inviscid stability of multidimensional planar shock fronts, Indiana Univ. Math. J. 48 (1999), no.3, 937-992. 